\title{
Minkowski valuations on lattice polytopes
}

\author{
Károly J. Böröczky and Monika Ludwig
}

\begin{abstract}
A complete classification is established of Minkowski valuations on lattice polytopes that intertwine the special linear group over the integers and are translation invariant. In the contravariant case, the only such valuations are multiples of projection bodies. In the equivariant case, the only such valuations are generalized difference bodies combined with multiples of the newly defined discrete Steiner point.
\end{abstract}

2000 AMS subject classification: 52B20, 52B45

\section{Introduction and statement of results}

Two classification theorems were critical in the beginning of the theory of valuations on convex sets: first, the Hadwiger theorem 23 for valuations on convex bodies (that is, compact convex sets) in $\mathbb{R}^{n}$ and second, the Betke \& Kneser theorem [11 for valuations on lattice polytopes (that is, convex polytopes with vertices in $\mathbb{Z}^{n}$ ). In recent years, numerous classification results were established for valuations defined on convex bodies (see, for example, $[4,5,9,20,21,29,32,33,45,52]$ and $[23,26,40,42$ for more information). In particular, such results were obtained for convex-body valued valuations (see, for example, [1 3, 6, 18, 19, 27, 28, 31, 43, 44, 49 51]). The aim of this article is to establish classification results for convex-body valued valuations defined on lattice polytopes. The question leads us to define and classify the discrete Steiner point.

A function z defined on a family $\mathcal{F}$ of subsets of $\mathbb{R}^{n}$ with values in an abelian group (or more generally, an abelian monoid) is a valuation if

$$
\mathrm{z}(P)+\mathrm{z}(Q)=\mathrm{z}(P \cup Q)+\mathrm{z}(P \cap Q)
$$

whenever $P, Q, P \cup Q, P \cap Q \in \mathcal{F}$ and $\mathrm{z}(\emptyset)=0$. 
In the Hadwiger theorem, $\mathcal{F}$ is the family, $\mathcal{K}\left(\mathbb{R}^{n}\right)$, of convex bodies and important further results regard the family, $\mathcal{P}\left(\mathbb{R}^{n}\right)$, of convex polytopes in $\mathbb{R}^{n}$. In both cases, the spaces are equipped with the topology coming from the Hausdorff metric. A functional $\mathrm{z}: \mathcal{K}\left(\mathbb{R}^{n}\right) \rightarrow \mathbb{R}$ is rigid motion invariant, if it is translation invariant and invariant with respect to orthogonal linear transformations.

Theorem 1 (Hadwiger [23]). A functional $\mathrm{z}: \mathcal{K}\left(\mathbb{R}^{n}\right) \rightarrow \mathbb{R}$ is a continuous and rigid motion invariant valuation if and only if there exist constants $c_{0}$, $c_{1}, \ldots, c_{n} \in \mathbb{R}$ such that

$$
\mathrm{z}(K)=c_{0} V_{0}(K)+\cdots+c_{n} V_{n}(K)
$$

for every $K \in \mathcal{K}\left(\mathbb{R}^{n}\right)$.

Here $V_{0}(K), \ldots, V_{n}(K)$ are the intrinsic volumes of $K \in \mathcal{K}\left(\mathbb{R}^{n}\right)$. An elegant proof of this result is due to Klain [25] (or see [26, 48]).

In the Betke \& Kneser theorem (and in this article), $\mathcal{F}$ is the family, $\mathcal{P}\left(\mathbb{Z}^{n}\right)$, of lattice polytopes. A functional $\mathrm{z}: \mathcal{P}\left(\mathbb{Z}^{n}\right) \rightarrow \mathbb{R}$ is called translation invariant if $\mathrm{z}(P+x)=\mathrm{z}(P)$ for $x \in \mathbb{Z}^{n}$ and $P \in \mathcal{P}\left(\mathbb{Z}^{n}\right)$. It is $\mathrm{SL}_{n}(\mathbb{Z})$ invariant if $\mathrm{z}(\phi P)=\mathrm{z}(P)$ for $\phi \in \mathrm{SL}_{n}(\mathbb{Z})$ and $P \in \mathcal{P}\left(\mathbb{Z}^{n}\right)$, where $\mathrm{SL}_{n}(\mathbb{Z})$ is the special linear group over $\mathbb{Z}$, that is, the group of invertible $n \times n$ matrices with integer coefficients and determinant 1 . We remark that Betke \& Kneser formulated their theorem for unimodularly invariant valuations (that is, also admitting matrices with determinant -1 ) but that their proof also establishes the following result.

Theorem 2 (Betke \& Kneser [11]). A functional $\mathrm{z}: \mathcal{P}\left(\mathbb{Z}^{n}\right) \rightarrow \mathbb{R}$ is an $\mathrm{SL}_{n}(\mathbb{Z})$ and translation invariant valuation if and only if there exist constants $c_{0}, c_{1}, \ldots, c_{n} \in \mathbb{R}$ such that

$$
\mathrm{z}(P)=c_{0} L_{0}(P)+\cdots+c_{n} L_{n}(P)
$$

for every $P \in \mathcal{P}\left(\mathbb{Z}^{n}\right)$.

Here $L_{0}(P), \ldots, L_{n}(P)$ are the Ehrhart functionals of $P \in \mathcal{P}\left(\mathbb{Z}^{n}\right)$, that is, the coefficients of the Ehrhart polynomial (see Section 3 for the definition). 
An operator $\mathrm{Z}: \mathcal{F} \rightarrow \mathcal{K}\left(\mathbb{R}^{n}\right)$ is called a Minkowski valuation if $\mathrm{Z}$ satisfies (1) and addition on $\mathcal{K}\left(\mathbb{R}^{n}\right)$ is Minkowski addition; that is,

$$
K+L=\{x+y: x \in K, y \in L\} .
$$

An operator $\mathrm{Z}: \mathcal{F} \rightarrow \mathcal{K}\left(\mathbb{R}^{n}\right)$ is called $\mathrm{SL}_{n}(\mathbb{R})$ equivariant if $\mathrm{Z}(\phi P)=\phi \mathrm{Z} P$ for $\phi \in \mathrm{SL}_{n}(\mathbb{R})$ and $P \in \mathcal{F}$. Define $\mathrm{SL}_{n}(\mathbb{Z})$ equivariance of operators on $\mathcal{P}\left(\mathbb{Z}^{n}\right)$ analogously. In recent years, $\mathrm{SL}_{n}(\mathbb{R})$ equivariant operators on convex bodies and the associated inequalities have attracted increased interest (see, for example, [13, 22, 34,36, 38] $)$. For valuations $\mathrm{Z}: \mathcal{P}\left(\mathbb{R}^{n}\right) \rightarrow \mathcal{K}\left(\mathbb{R}^{n}\right)$ that are $\mathrm{SL}_{n}(\mathbb{R})$ equivariant and translation invariant, a complete classification has been established. Let $n \geq 2$.

Theorem 3 ([30]). An operator $\mathrm{Z}: \mathcal{P}\left(\mathbb{R}^{n}\right) \rightarrow \mathcal{K}\left(\mathbb{R}^{n}\right)$ is an $\mathrm{SL}_{n}(\mathbb{R})$ equivariant and translation invariant Minkowski valuation if and only if there exists a constant $c \geq 0$ such that

$$
\mathrm{Z} P=c(P-P)
$$

for every $P \in \mathcal{P}\left(\mathbb{R}^{n}\right)$.

The operator $P \mapsto P-P=\{x-y: x, y \in P\}$ assigns to $P$ its difference body. We remark that no complete analogue of Hadwiger's theorem for Minkowski valuations (that is, no complete classification of rotation equivariant and translation invariant Minkowski valuations) has been established. It follows from, for example, [49] that the set of such valuations does not depend on only finitely many parameters.

The aim of this article is to classify Minkowski valuations on lattice polytopes. The following result is an analogue of Theorem 3. Let $n \geq 2$.

Theorem 4. An operator $\mathrm{Z}: \mathcal{P}\left(\mathbb{Z}^{n}\right) \rightarrow \mathcal{K}\left(\mathbb{R}^{n}\right)$ is an $\mathrm{SL}_{n}(\mathbb{Z})$ equivariant and translation invariant Minkowski valuation if and only if there exist constants $a, b \geq 0$ such that

$$
\mathrm{Z} P=a\left(P-\ell_{1}(P)\right)+b\left(-P+\ell_{1}(P)\right)
$$

for every $P \in \mathcal{P}\left(\mathbb{Z}^{n}\right)$.

Here for a lattice polytope $P$, the point $\ell_{1}(P)$ is its discrete Steiner point that is introduced in this paper. 
The discrete Steiner point is defined in Section 3 as the one-homogeneous part of the Ehrhart expansion of the discrete moment vector

$$
\ell(P)=\sum_{x \in P \cap \mathbb{Z}^{n}} x
$$

That such an expansion exists follows from results by McMullen [39]. The discrete moment vector plays for $\mathrm{SL}_{n}(\mathbb{Z})$ equivariant vector-valued valuations on $\mathcal{P}\left(\mathbb{Z}^{n}\right)$ a role similar to that of the moment vector

$$
m_{n+1}(K)=\int_{K} x \mathrm{~d} x
$$

for rigid motion equivariant valuations on $\mathcal{K}\left(\mathbb{R}^{n}\right)$.

The discrete Steiner point is characterized in the following result, where $\mathrm{z}: \mathcal{P}\left(\mathbb{Z}^{n}\right) \rightarrow \mathbb{R}^{n}$ is called translation equivariant if $\mathrm{z}(P+x)=\mathrm{z}(P)+x$ for $x \in \mathbb{Z}^{n}$ and $P \in \mathcal{P}\left(\mathbb{Z}^{n}\right)$.

Theorem 5. A function $\mathrm{z}: \mathcal{P}\left(\mathbb{Z}^{n}\right) \rightarrow \mathbb{R}^{n}$ is an $\mathrm{SL}_{n}(\mathbb{Z})$ and translation equivariant valuation if and only if $\mathrm{z}=\ell_{1}$.

Theorem 5 corresponds to the following characterization of the classical Steiner point $m_{1}$, which is the one-homogeneous part of the Steiner expansion of the moment vector (see Section 2 for the definition).

Theorem 6 (Schneider [47]). A function $\mathrm{z}: \mathcal{K}\left(\mathbb{R}^{n}\right) \rightarrow \mathbb{R}^{n}$ is a continuous and rigid motion equivariant valuation if and only if $\mathrm{z}=m_{1}$.

A function $\mathrm{z}: \mathcal{P}\left(\mathbb{Z}^{n}\right) \rightarrow \mathbb{R}^{n}$ is called additive if $\mathrm{z}(P+Q)=\mathrm{z}(P)+\mathrm{z}(Q)$ for $P, Q \in \mathcal{P}\left(\mathbb{Z}^{n}\right)$. The discrete Steiner point is also characterized in the following result.

Theorem 7. A function $\mathrm{z}: \mathcal{P}\left(\mathbb{Z}^{n}\right) \rightarrow \mathbb{R}^{n}$ is $\mathrm{SL}_{n}(\mathbb{Z})$ and translation equivariant and additive if and only if $\mathrm{z}=\ell_{1}$.

Theorem 7 corresponds to the following characterization of the classical Steiner point.

Theorem 8 (Schneider [46]). A function $\mathrm{z}: \mathcal{K}\left(\mathbb{R}^{n}\right) \rightarrow \mathbb{R}^{n}$ is continuous, rigid motion equivariant and additive if and only if $\mathrm{z}=m_{1}$. 
For operators mapping $\mathcal{P}\left(\mathbb{Z}^{n}\right)$ to $\mathcal{P}\left(\mathbb{Z}^{n}\right)$, we obtain the following result. Write LCM for the least common multiple and let $n \geq 2$.

Theorem 9. An operator $\mathrm{Z}: \mathcal{P}\left(\mathbb{Z}^{n}\right) \rightarrow \mathcal{P}\left(\mathbb{Z}^{n}\right)$ is an $\mathrm{SL}_{n}(\mathbb{Z})$ equivariant and translation invariant Minkowski valuation if and only if there exist integers $a, b \geq 0$ with $b-a \in \operatorname{LCM}(2, \ldots, n+1) \mathbb{Z}$ such that

$$
\mathrm{Z} P=a\left(P-\ell_{1}(P)\right)+b\left(-P+\ell_{1}(P)\right)
$$

for every $P \in \mathcal{P}\left(\mathbb{Z}^{n}\right)$.

An operator $\mathrm{Z}: \mathcal{F} \rightarrow \mathcal{K}\left(\mathbb{R}^{n}\right)$ is called $\mathrm{SL}_{n}(\mathbb{R})$ contravariant if $\mathrm{Z}(\phi P)=$ $\phi^{-t} \mathrm{Z} P$ for $\phi \in \mathrm{SL}_{n}(\mathbb{R})$ and $P \in \mathcal{F}$, where $\phi^{-t}$ is the inverse of the transpose of $\phi$. Define $\mathrm{SL}_{n}(\mathbb{Z})$ contravariance of operators on $\mathcal{P}\left(\mathbb{Z}^{n}\right)$ analogously. In recent years, $\mathrm{SL}_{n}(\mathbb{R})$ contravariant operators on convex bodies and the associated inequalities have attracted increased interest (see, for example, $[12,22,34,37])$. For $\mathrm{SL}_{n}(\mathbb{R})$ contravariant Minkowski valuations on $\mathcal{P}\left(\mathbb{R}^{n}\right)$, a complete classification has been established. Let $n \geq 2$.

Theorem 10 ([30]). An operator $\mathrm{Z}: \mathcal{P}\left(\mathbb{R}^{n}\right) \rightarrow \mathcal{K}\left(\mathbb{R}^{n}\right)$ is an $\mathrm{SL}_{n}(\mathbb{R})$ contravariant and translation invariant Minkowski valuation if and only if there exists a constant $c \geq 0$ such that

$$
\mathrm{Z} P=c \Pi P
$$

for every $P \in \mathcal{P}\left(\mathbb{R}^{n}\right)$.

Here $\Pi P$ is the projection body of $P$ (see Section 2 for the definition).

For operators on lattice polytopes, we obtain the following result.

Theorem 11. (i) For $n=2$, an operator $\mathrm{Z}: \mathcal{P}\left(\mathbb{Z}^{2}\right) \rightarrow \mathcal{K}\left(\mathbb{R}^{2}\right)$ is an $\mathrm{SL}_{2}(\mathbb{Z})$ contravariant and translation invariant Minkowski valuation if and only if there exist constants $a, b \geq 0$ such that

$$
\mathrm{Z} P=a \rho_{\pi / 2}\left(P-\ell_{1}(P)\right)+b \rho_{\pi / 2}\left(-P+\ell_{1}(P)\right)
$$

for every $P \in \mathcal{P}\left(\mathbb{Z}^{2}\right)$.

(ii) For $n \geq 3$, an operator $\mathrm{Z}: \mathcal{P}\left(\mathbb{Z}^{n}\right) \rightarrow \mathcal{K}\left(\mathbb{R}^{n}\right)$ is an $\mathrm{SL}_{n}(\mathbb{Z})$ contravariant and translation invariant Minkowski valuation if and only if there exists a constant $c \geq 0$ such that

$$
\mathrm{Z} P=c \Pi P
$$

for every $P \in \mathcal{P}\left(\mathbb{Z}^{n}\right)$.

Here $\rho_{\pi / 2}$ denotes the rotation by an angle $\pi / 2$ in $\mathbb{R}^{2}$. Note that for $n=2$, the projection body is obtained from the difference body by applying $\rho_{\pi / 2}$. 
For operators mapping $\mathcal{P}\left(\mathbb{Z}^{n}\right)$ to $\mathcal{P}\left(\mathbb{Z}^{n}\right)$, we obtain the following result.

Theorem 12. (i) For $n=2$, an operator $\mathrm{Z}: \mathcal{P}\left(\mathbb{Z}^{2}\right) \rightarrow \mathcal{P}\left(\mathbb{Z}^{2}\right)$ is an $\mathrm{SL}_{2}(\mathbb{Z})$ contravariant and translation invariant Minkowski valuation if and only if there exist integers $a, b \geq 0$ with $b-a \in 6 \mathbb{Z}$ such that

$$
\mathrm{Z} P=a \rho_{\pi / 2}\left(P-\ell_{1}(P)\right)+b \rho_{\pi / 2}\left(-P+\ell_{1}(P)\right)
$$

for every $P \in \mathcal{P}\left(\mathbb{Z}^{2}\right)$.

(ii) For $n \geq 3$, an operator $\mathrm{Z}: \mathcal{P}\left(\mathbb{Z}^{n}\right) \rightarrow \mathcal{P}\left(\mathbb{Z}^{n}\right)$ is an $\mathrm{SL}_{n}(\mathbb{Z})$ contravariant and translation invariant Minkowski valuation if and only if there exists a constant $c \geq 0$ with $c \in(n-1) ! \mathbb{Z}$ such that

$$
\mathrm{Z} P=c \Pi P
$$

for every $P \in \mathcal{P}\left(\mathbb{Z}^{n}\right)$.

\section{Preliminaries}

We collect notation and results on convex bodies, valuations and lattice polytopes. General references are Schneider [48, Gruber [17], Barvinok [7] and Beck \& Robins [8].

Every convex body $K \in \mathcal{K}\left(\mathbb{R}^{n}\right)$ is determined by its support function,

$$
h(K, v)=\max \{v \cdot x: x \in K\}
$$

for $v \in \mathbb{R}^{n}$, where $v \cdot x$ is the inner product of $v, x \in \mathbb{R}^{n}$. Note that for $v \in \mathbb{R}^{n}$ we have

$$
h(K+L, v)=h(K, v)+h(L, v) .
$$

Support functions of convex bodies are sublinear, that is, they are convex and positively homogeneous of degree 1, and every sublinear function is the support function of a convex body in $\mathbb{R}^{n}$.

For $M \subset \mathbb{R}^{n}$, we denote the affine hull by aff $M$ and the dimension (that is, the dimension of aff $M$ ) by $\operatorname{dim}(M)$. Define the centroid of a $k$-dimensional set $M$ with positive $k$-dimensional volume $V_{k}(M)$ by

$$
\operatorname{cen}(M)=\frac{1}{V_{k}(M)} \int_{M} x \mathrm{~d} H^{k}(x),
$$

where $H^{k}$ is the $k$-dimensional Hausdorff measure. We denote the convex hull of $x_{1}, \ldots, x_{k} \in \mathbb{R}^{n}$ by $\left[x_{1}, \ldots, x_{k}\right]$. 


\subsection{Minkowski summands}

Understanding the structure of summands is critical to our argument. A convex body $L$ is a summand of a convex body $K$ if there exists a convex body $M$ such that $K=L+M$.

For $L \in \mathcal{K}\left(\mathbb{R}^{n}\right)$ and $v \in \mathbb{R}^{n} \backslash\{o\}$, we define the face of $L$ having $v$ as one of its normal vectors by

$$
F(L, v)=\{x \in L: v \cdot x=h(L, v)\} .
$$

It follows that if $L, M \in \mathcal{K}\left(\mathbb{R}^{n}\right)$ and $s, t \geq 0$, then

$$
F(s L+t M, v)=s F(L, v)+t F(M, v) .
$$

Note that if $K=L+M$ is a polytope, then so are $L$ and $M$. Also note that the only summands of a simplex $S$ are translates of $t S$ with $t \in[0,1]$ and that a summand of a direct sum of two convex bodies is the direct sum of summands of these bodies (see [48, Section 3.2]). Combined with (2) this implies the following.

Lemma 13. Let $S$ be a simplex and $R$ a convex body with $\operatorname{dim}(S+R)=$ $\operatorname{dim}(S)+\operatorname{dim}(R)$. If a convex body $L$ is a summand of $S+R$, then there exist $t \in[0,1]$ and $R^{\prime} \subset$ aff $R$ such that $L$ is a translate of $t S+R^{\prime}$.

\subsection{Projection bodies}

For $u \in \mathbb{S}^{n-1}$ (where $\mathbb{S}^{n-1}$ is the $(n-1)$-dimensional unit sphere), let $\pi_{u}$ denote the orthogonal projection to the subspace orthogonal to $u$. For $K \in \mathcal{K}\left(\mathbb{R}^{n}\right)$, the projection body $\Pi K$ is defined by

$$
h(\Pi K, u)=\left|\pi_{u} K\right|
$$

for $u \in \mathbb{S}^{n-1}$, where $|\cdot|$ denotes $(n-1)$-dimensional volume. Note that if $P$ is an $n$-dimensional polytope in $\mathbb{R}^{n}$ with facets (that is, $(n-1)$-dimensional faces) $F_{1}, \ldots, F_{m}$, and corresponding facet normals (that is, exterior unit normals) $u_{1}, \ldots, u_{m}$, then

$$
\Pi P=\frac{1}{2} \sum_{i=1}^{m}\left|F_{i}\right|\left[-u_{i}, u_{i}\right]
$$


The Minkowski relation states that

$$
\sum_{i=1}^{m}\left|F_{i}\right| u_{i}=o
$$

See [16] for more information on projection bodies.

\subsection{The Steiner point}

The intrinsic volumes that are characterized in Hadwiger's theorem are the coefficients of the Steiner polynomial, that is,

$$
V_{n}\left(K+s B^{n}\right)=\sum_{j=0}^{n} s^{n-j} v_{n-j} V_{j}(K),
$$

where $B^{n}$ is the $n$-dimensional Euclidean unit ball and $v_{j}$ is the $j$-dimensional volume of the $j$-dimensional Euclidean unit ball. The corresponding expansion for the moment vector is

$$
m_{n+1}\left(K+s B^{n}\right)=\sum_{j=1}^{n+1} s^{n+1-j} v_{n+1-j} m_{j}(K) .
$$

The Steiner point, $m_{1}(K)$, can also be represented as

$$
m_{1}(K)=\frac{1}{v_{n}} \int_{\mathbb{S}^{n-1}} u h(K, u) \mathrm{d} H^{n-1}(u) .
$$

For more information on Steiner points, see [48, Section 5.4].

\subsection{The inclusion-exclusion principle}

Betke (unpublished) and McMullen [41] extended (11) to an inclusion-exclusion principle. Let $\mathbb{G}$ be an abelian group.

Theorem 14 (McMullen [41]). If $\mathrm{z}: \mathcal{P}\left(\mathbb{Z}^{n}\right) \rightarrow \mathbb{G}$ is a valuation, then for lattice polytopes $P_{1}, \ldots, P_{m}$,

$$
\mathrm{z}\left(P_{1} \cup \cdots \cup P_{m}\right)=\sum_{\substack{1 \leq i_{1}<\cdots<i_{k} \leq m \\ 1 \leq k \leq m}}(-1)^{k-1} \mathrm{z}\left(P_{i_{1}} \cap \cdots \cap P_{i_{k}}\right)
$$

whenever $P_{1} \cup \cdots \cup P_{m}$ and all intersections of the form $P_{i_{1}} \cap \cdots \cap P_{i_{k}}$ are lattice polytopes. 
The inclusion-exclusion formula is actually needed for cell decompositions in this paper. We call a dissection of the $n$-dimensional lattice polytope $Q$ into $n$-dimensional lattice polytopes $P_{1}, \ldots, P_{m}$ a cell decomposition if $P_{i} \cap P_{j}$ is either empty or a common face of $P_{i}$ and $P_{j}$ for any $1 \leq i<j \leq m$. The faces of the cell decomposition are the faces of all $P_{i}$ for $i=1, \ldots, m$. Let int $Q$ denote the interior of $Q$.

Corollary 15. If $\mathrm{z}: \mathcal{P}\left(\mathbb{Z}^{n}\right) \rightarrow \mathbb{G}$ is a valuation and $Q$ an $n$-dimensional lattice polytope, then

$$
\mathrm{z}(Q)=\sum_{\substack{F \in \mathcal{F} \\ F \cap \operatorname{int} Q \neq \emptyset}}(-1)^{n-\operatorname{dim}(F)} \mathrm{z}(F)
$$

where $\mathcal{F}$ is the set of faces of a cell decomposition of $Q$ into lattice polytopes.

Proof. Let $\mathbf{1}_{P}$ be the characteristic function of $P \in \mathcal{P}\left(\mathbb{Z}^{n}\right)$ and $[\mathcal{P}]\left(\mathbb{Z}^{n}\right)$ the additive abelian group generated by characteristics functions of lattice polytopes. McMullen [41, Theorem 8.1(c)] established the following form of the inclusion-exclusion principle. For any valuation $\mathrm{z}: \mathcal{P}\left(\mathbb{Z}^{n}\right) \rightarrow \mathbb{G}$ there exists a homomorphism $[\mathrm{z}]:[\mathcal{P}]\left(\mathbb{Z}^{n}\right) \rightarrow \mathbb{G}$ such that $\mathrm{z}(P)=[\mathrm{z}]\left(\mathbf{1}_{P}\right)$ for $P \in \mathcal{P}\left(\mathbb{Z}^{n}\right)$.

Hence it suffices to show that

$$
\mathbf{1}_{Q}=\sum_{\substack{F \in \mathcal{F} \\ F \cap \operatorname{int} Q \neq \emptyset}}(-1)^{n-\operatorname{dim}(F)} \mathbf{1}_{F} .
$$

Clearly, (5) is true on the complement of $Q$. For $x \in Q$, let $\mathcal{S}_{x}$ be the set of faces of $\mathcal{F}$ that have non-empty intersection with $\operatorname{int} Q$ and contain $x$ and let $\mathcal{L}_{x}$ be the boundary complex of the set underlying $\mathcal{S}_{x}$. For a family of polytopes $\mathcal{G}$, define $\chi(\mathcal{G})$ as the number of even dimensional polytopes minus the number of odd dimensional polytopes in $\mathcal{G}$. For the cell complex $\mathcal{S}_{x} \cup \mathcal{L}_{x}$, we obtain the Euler characteristic and $\chi\left(\mathcal{S}_{x}\right)+\chi\left(\mathcal{L}_{x}\right)=1$ since the underlying set is homeomorphic to an $n$-dimensional ball. This also implies $\chi\left(\mathcal{L}_{x}\right)=1-(-1)^{n}$. Hence

$$
\sum_{F \in \mathcal{S}_{x}}(-1)^{n-\operatorname{dim}(F)}=(-1)^{n} \chi\left(\mathcal{S}_{x}\right)=(-1)^{n}\left(1-\chi\left(\mathcal{L}_{x}\right)\right),
$$

which proves (5). 


\subsection{Triangulations}

Write $e_{1}, \ldots, e_{n}$ for the standard orthonormal basis of $\mathbb{R}^{n}$, which generates $\mathbb{Z}^{n}$, and write $o$ for the origin. Define $T_{0}=\{o\}$ and $T_{i}=\left[o, e_{1}, \ldots, e_{i}\right]$ for $i=1, \ldots, n$. We call a lattice simplex basic if it is obtained from $T_{i}$ for some $i=0, \ldots, n$ by a map from $\mathrm{SL}_{n}(\mathbb{Z})$ followed by a translation.

In addition, let $[0,1]^{i}=\left[o, e_{1}\right]+\cdots+\left[o, e_{i}\right]$ be the standard $i$-dimensional unit cube. One of the main ideas in this paper is to relate $\mathrm{Z} T_{n}$ and $\mathrm{Z}[0,1]^{n}$ for a Minkowski valuation $\mathrm{Z}$ on $\mathcal{P}\left(\mathbb{Z}^{n}\right)$. In order to do that, we write $R_{n}$ for the convex hull of all vertices of $[0,1]^{n}$ but $o$. Hence $R_{n} \cup T_{n}=[0,1]^{n}$ and $R_{n} \cap T_{n}=\left[e_{1}, \ldots, e_{n}\right]$. Since $\mathrm{Z}$ is a valuation, we get

$$
\mathrm{Z}[0,1]^{n}+\mathrm{Z}\left[e_{1}, \ldots, e_{n}\right]=\mathrm{Z} T_{n}+\mathrm{Z} R_{n} .
$$

In the case of $\mathrm{SL}_{n}(\mathbb{Z})$ equivariant and translation invariant Minkowski valuations, we also need another specific cell decomposition involving $T_{n}$. For the prism $\widetilde{T}_{n-1}=T_{n-1}+\left[0, e_{n}\right]$, it will be useful to consider a cell de-

composition of $\widetilde{T}_{n-1}$ into $n$ simplices $S_{1}, \ldots, S_{n}$. Setting $e_{0}=o$, we define $S_{1}=T_{n}$ and

$$
S_{i}=\left[e_{0}+e_{n}, \ldots, e_{i-1}+e_{n}, e_{i-1}, \ldots, e_{n-1}\right] \text { for } i=2, \ldots, n .
$$

Note that each $S_{i}$ is basic and that $\operatorname{dim}\left(S_{i} \cap S_{j}\right)=n-1$ for $i<j$ if and only if $j=i+1$ (see, for example, [24, Section 2.1]).

We also require the following result (see, for example, [14, Section 6.3]).

Lemma 16. There exists a triangulation of $[0,1]^{n}$ into $n$ ! basic simplices using only the vertices of the cube such that $T_{n}$ is one of these simplices.

\subsection{The Betke \& Kneser theorem}

Betke [10] and Betke \& Kneser [11] proved Theorem 2] by using suitable dissections and complementations of lattice polytopes by lattice simplices.

Proposition 17 (Betke \& Kneser [1]]). For every lattice polytope $P \in \mathcal{P}\left(\mathbb{Z}^{n}\right)$ there exist basic simplices $S_{1}, \ldots, S_{m}$ and integers $k_{1}, \ldots, k_{m}$ such that

$$
\mathrm{z}(P)=\sum_{i=1}^{m} k_{i} \mathrm{z}\left(S_{i}\right)
$$

for all valuations $\mathrm{z}$ on $\mathcal{P}\left(\mathbb{Z}^{n}\right)$ with values in an abelian group. 
The following statement is a direct consequence of this proposition.

Corollary 18. If $\mathrm{Z}, \mathrm{Z}^{\prime}: \mathcal{P}\left(\mathbb{Z}^{n}\right) \rightarrow \mathcal{K}\left(\mathbb{R}^{n}\right)$ are $\mathrm{SL}_{n}(\mathbb{Z})$ equivariant (or $\mathrm{SL}_{n}(\mathbb{Z})$ contravariant) and translation invariant Minkowski valuations such that

$$
\mathrm{Z} T_{i}=\mathrm{Z}^{\prime} T_{i} \text { for } i=0, \ldots, n,
$$

then $\mathrm{Z}=\mathrm{Z}^{\prime}$ on $\mathcal{P}\left(\mathbb{Z}^{n}\right)$.

Proof. For $v \in \mathbb{R}^{n}$, the functions $P \mapsto h(\mathrm{Z} P, v)$ and $P \mapsto h\left(\mathrm{Z}^{\prime} P, v\right)$ are realvalued valuations on $\mathcal{P}\left(\mathbb{Z}^{n}\right)$. For $P \in \mathcal{P}\left(\mathbb{Z}^{n}\right)$, Proposition 17 implies that there are basic simplices $S_{1}, \ldots, S_{m}$ and integers $k_{1}, \ldots, k_{m}$ such that

$$
h(\mathrm{Z} P, v)=\sum_{i=1}^{m} k_{i} h\left(\mathrm{Z} S_{i}, v\right) \text { and } h\left(\mathrm{Z}^{\prime} P, v\right)=\sum_{i=1}^{m} k_{i} h\left(\mathrm{Z}^{\prime} S_{i}, v\right) \text {. }
$$

Since $\mathrm{Z}$ and $\mathrm{Z}^{\prime}$ are $\mathrm{SL}_{n}(\mathbb{Z})$ equivariant (or $\mathrm{SL}_{n}(\mathbb{Z})$ contravariant) and translation invariant, (8) implies that $\mathrm{Z}=\mathrm{Z}^{\prime}$ on $\mathcal{P}\left(\mathbb{Z}^{n}\right)$.

\subsection{Translation invariant valuations}

We say that a valuation $\mathrm{z}$ with values in an abelian semigroup is homogeneous of degree $i \in \mathbb{N}$ if $\mathrm{z}(k P)=k^{i} \mathrm{z}(P)$ for $k \in \mathbb{N}$ and $P \in \mathcal{P}\left(\mathbb{Z}^{n}\right)$, where $\mathbb{N}$ denotes the set of non-negative integers.

McMullen [39] established the following theorem under the assumption of the inclusion-exclusion principle contained in Theorem 14, which he later established in [41].

Theorem 19 (McMullen). If $\mathrm{z}: \mathcal{P}\left(\mathbb{Z}^{n}\right) \rightarrow \mathbb{R}$ is a translation invariant valuation, then $\mathrm{z}(k P)$ is a polynomial in $k \in \mathbb{N}$ of degree $\operatorname{dim}(P)$ for every $P \in \mathcal{P}\left(\mathbb{Z}^{n}\right)$.

As an application of Theorem 19, we consider Minkowski valuations. The following construction goes back to [30].

Lemma 20. Let $\mathrm{Z}: \mathcal{P}\left(\mathbb{Z}^{n}\right) \rightarrow \mathcal{K}\left(\mathbb{R}^{n}\right)$ be a translation invariant Minkowski valuation. For $P \in \mathcal{P}\left(\mathbb{Z}^{n}\right)$, there exists a convex body

$$
\mathrm{Z}_{n} P=\lim _{k \rightarrow \infty} \frac{\mathrm{Z}(k P)}{k^{n}}
$$

and $\mathrm{Z}_{n}$ is a Minkowski valuation on $\mathcal{P}\left(\mathbb{Z}^{n}\right)$, which is homogeneous of degree $n$. If $\mathrm{Z}$ is $\mathrm{SL}_{n}(\mathbb{Z})$ equivariant or $\mathrm{SL}_{n}(\mathbb{Z})$ contravariant, then so is $\mathrm{Z}_{n}$. 
Proof. For $v \in \mathbb{R}^{n}$, the function $P \mapsto h(\mathrm{Z} P, v)$ is a real-valued valuation on $\mathcal{P}\left(\mathbb{Z}^{n}\right)$, which is translation invariant as $\mathrm{Z}$ is translation invariant. By Theorem 19, there exist coefficients $c_{i}(P, v) \in \mathbb{R}, i=0, \ldots, n$, for $v \in \mathbb{R}^{n}$ and $P \in \mathcal{P}\left(\mathbb{Z}^{n}\right)$ such that

$$
h(\mathrm{Z}(k P), v)=\sum_{i=0}^{n} c_{i}(P, v) k^{i} \text { for } k \in \mathbb{N} .
$$

Hence the limit $c_{n}(P, v)=\lim _{k \rightarrow \infty} h(\mathrm{Z}(k P), v) / k^{n}$ exists for $v \in \mathbb{R}^{n}$ and $c_{n}(P, \cdot)$ is a sublinear function on $\mathbb{R}^{n}$. Therefore $c_{n}(P, \cdot)$ is the support function of a convex body, which we call $\mathrm{Z}_{n} P$. Since $\mathrm{Z}$ is a Minkowski valuation, so is $Z_{n}$. In addition, for fixed $v$, the function $P \mapsto c_{n}(P, v)$ is homogeneous of degree $n$ in $P$ by Theorem $\left[19\right.$. Thus the same holds for $Z_{n}$. The equivariance follows immediately from the definition.

\subsection{Transforming into a regular simplex}

Because of Corollary 18, we concentrate on determining $\mathrm{Z} T_{n}$ in the proof of Theorems 4 and 11. We will make extensive use of the symmetries of $Z T_{n}$.

Let $\mathrm{GL}_{n}(\mathbb{R})$ denote the group of general linear transformations on $\mathbb{R}^{n}$. We write $T=T_{n}$ and set $\bar{T}=T-\operatorname{cen}(T)$, where $\operatorname{cen}(T)$ is the centroid of $T$. We fix a transformation $\alpha \in \mathrm{GL}_{n}(\mathbb{R})$ such that $\alpha \bar{T}$ is the regular simplex $T_{*}$ of circumradius one,

$$
\alpha \bar{T}=\left[v_{0}, \ldots, v_{n}\right]=T_{*},
$$

where $v_{0}=-\operatorname{cen}(\alpha T)$ and $v_{i}=v_{0}+\alpha e_{i}$ for $i=1, \ldots, n$. Let $\operatorname{Sym}\left(T_{*}\right)$ denote the group of orientation preserving isometries of the regular simplex $T_{*}$.

Note that

$$
v_{i} \cdot v_{j}=\left\{\begin{aligned}
1 & \text { if } i=j \\
-\frac{1}{n} & \text { if } i \neq j
\end{aligned}\right.
$$

We set

$$
w_{m}=v_{0}+\cdots+v_{m} \text { for } m=0, \ldots, n-1
$$

and obtain

$$
\begin{array}{r}
F\left(T_{*}, w_{m}\right)=\left[v_{0}, \ldots, v_{m}\right], \\
F\left(T_{*},-w_{m}\right)=\left[v_{m+1}, \ldots, v_{n}\right],
\end{array}
$$


and for $m=1, \ldots, n-1$,

$$
\begin{aligned}
F\left(\left[v_{1}, \ldots, v_{n}\right], w_{m}\right) & =\left[v_{1}, \ldots, v_{m}\right], \\
F\left(\left[v_{1}, \ldots, v_{n}\right],-w_{m}\right) & =\left[v_{m+1}, \ldots, v_{n}\right] .
\end{aligned}
$$

Note that all faces of $T_{*}$ are obtained as image of $F\left(T_{*}, w_{m}\right)$ by suitable maps from $\operatorname{Sym}\left(T_{*}\right)$ for all $m=0, \ldots, n-1$.

In the equivariant case, generalized difference bodies are important for us. A facet normal of the polytope $T_{*}-T_{*}$ is a positive multiple of $\sum_{i \in I} v_{i}$ where $I$ is a proper subset of $\{0, \ldots, n\}$. Because of (2), the same holds true for the facet normals of $s T_{*}-t T_{*}$ with $s, t \geq 0$. Since (in any dimension)

$$
s T_{*}-t T_{*}=\left[s v_{i}-t v_{j}: i, j=0, \ldots, n, i \neq j\right],
$$

we get

$$
s T_{*}-t T_{*}=\left[\rho F\left(s T_{*}-t T_{*}, w_{m}\right): \rho \in \operatorname{Sym}\left(T_{*}\right)\right]
$$

for each $m=0, \ldots, n-1$. Indeed, the right side is clearly contained in $s T_{*}-t T_{*}$ and it follows from (11) that each $s v_{i}-t v_{j}$ for $i \neq j$ is contained in the right side.

\section{The discrete Steiner point}

For $P \in \mathcal{P}\left(\mathbb{Z}^{n}\right)$, let $L(P)$ denote the number of lattice points in $P$, that is,

$$
L(P)=\sum_{x \in P \cap \mathbb{Z}^{n}} 1 .
$$

The function $L: \mathcal{P}\left(\mathbb{Z}^{n}\right) \rightarrow \mathbb{Z}$ is a valuation that is invariant with respect to unimodular linear transformations. In addition, if $z \in \mathbb{Z}^{n}$, then

$$
L(P+z)=L(P)
$$

that is, $L$ is translation invariant. We call a function that is invariant with respect to unimodular linear transformations and translations by integer vectors unimodularly invariant. 
Ehrhart [15] established the following result.

Theorem 21 (Ehrhart). There exist $L_{i}: \mathcal{P}\left(\mathbb{Z}^{n}\right) \rightarrow \mathbb{Q}$ for $i=0, \ldots, n$ such that

$$
L(k P)=\sum_{i=0}^{n} L_{i}(P) k^{i}
$$

for $k \in \mathbb{N}$ and $P \in \mathcal{P}\left(\mathbb{Z}^{n}\right)$. For each $i$, the functional $L_{i}$ is a unimodularly invariant valuation which is homogeneous of degree $i$.

Note that $L_{n}$ is the $n$-dimensional volume and $L_{0}$ is the Euler characteristic.

A special case of a more general result by McMullen [39, Theorem 6] implies

Theorem 22 (McMullen). If $P_{1}, \ldots, P_{m} \in \mathcal{P}\left(\mathbb{Z}^{n}\right)$, then the number of lattice points $L\left(k_{1} P_{1}+\cdots+k_{m} P_{m}\right)$ is a polynomial in $k_{1}, \ldots, k_{m} \in \mathbb{N}$.

From this, the following analogue of Remark 6.3.3 in Schneider [48] is obtained.

Corollary 23. The functional $L_{1}: \mathcal{P}\left(\mathbb{Z}^{n}\right) \rightarrow \mathbb{Q}$ is Minkowski additive.

Proof. For $P, Q \in \mathcal{P}\left(\mathbb{Z}^{n}\right)$ and $k, l \in \mathbb{N}$, by Theorem 22 we see that $L(k P+l Q)$ is a polynomial in $k$ and $l$. Considering the expression first as a function of $l$ when $k=0$ and second as a function of $k$ when $l=0$, we deduce that the linear term in $L(k P+l Q)$ is $L_{1}(P) k+L_{1}(Q) l$. In particular, the linear term in the one variable polynomial $L(k P+k Q)$ is $L_{1}(P) k+L_{1}(Q) k$ on the one hand and by Theorem 21 we get $L_{1}(P+Q) k$ on the other hand. Hence $L_{1}(P+Q)=L_{1}(P)+L_{1}(Q)$.

In analogy to (14), for $P \in \mathcal{P}\left(\mathbb{Z}^{n}\right)$, we define the discrete moment vector by

$$
\ell(P)=\sum_{x \in P \cap \mathbb{Z}^{n}} x
$$

The discrete moment vector $\ell: \mathcal{P}\left(\mathbb{Z}^{n}\right) \rightarrow \mathbb{Z}^{n}$ is a valuation that is equivariant with respect to unimodular linear transformations. In addition, if $z \in \mathbb{Z}^{n}$, then

$$
\ell(P+z)=\ell(P)+L(P) z .
$$

In particular, $\ell$ is not translation invariant or equivariant. In the terminology of [39], $\ell$ is an extended $\mathbb{Z}^{n}$-valuation. 
As a special case of a more general result by McMullen [39, Theorem 14] we obtain the following result.

Theorem 24 (McMullen). There exist $\ell_{i}: \mathcal{P}\left(\mathbb{Z}^{n}\right) \rightarrow \mathbb{Q}^{n}$ for $i=1, \ldots, n+1$ such that

$$
\ell(k P)=\sum_{i=1}^{n+1} \ell_{i}(P) k^{i}
$$

for $k \in \mathbb{N}$ and $P \in \mathcal{P}\left(\mathbb{Z}^{n}\right)$. For each $i$, the function $\ell_{i}$ is a valuation which is equivariant with respect to unimodular linear transformations and homogeneous of degree $i$.

We call $\ell_{1}(P)$ the discrete Steiner point of the lattice polytope $P$.

A special case of a more general result by McMullen [39, Theorem 14] implies the following result.

Theorem 25 (McMullen). If $P_{1}, \ldots, P_{m} \in \mathcal{P}\left(\mathbb{Z}^{n}\right)$, then the discrete moment vector $\ell\left(k_{1} P_{1}+\cdots+k_{m} P_{m}\right)$ is a polynomial in $k_{1}, \ldots, k_{m} \in \mathbb{N}$.

From this, we deduce as in Corollary 23 the following result.

Corollary 26. The functional $\ell_{1}: \mathcal{P}\left(\mathbb{Z}^{n}\right) \rightarrow \mathbb{Q}^{n}$ is additive.

In the next proposition, we collect some properties of the functional $\ell_{1}$. We require the following lemma.

Lemma 27. If $\mathrm{z}: \mathcal{P}\left(\mathbb{Z}^{n}\right) \rightarrow \mathbb{R}^{n}$ is an $\mathrm{SL}_{n}(\mathbb{Z})$ equivariant valuation, then

$$
\mathrm{z}\left((m+1) T_{m}-\operatorname{cen}\left((m+1) T_{m}\right)\right)=o .
$$

Proof. First, let $m=n$. Note that $\operatorname{cen}\left((n+1) T_{n}\right) \in \mathbb{Z}^{n}$. Set

$$
S_{n}=(n+1) T_{n}-\operatorname{cen}\left((n+1) T_{n}\right) .
$$

Since $\mathrm{z}$ is $\mathrm{SL}_{n}(\mathbb{Z})$ equivariant, we obtain from $\alpha^{-1} \rho \alpha S_{n}=S_{n}$ (with $\alpha$ defined in (9)) that

$$
\alpha \mathrm{z}\left(S_{n}\right)=\rho \alpha \mathrm{z}\left(S_{n}\right)
$$

for all $\rho \in \operatorname{Sym}\left(T_{*}\right)$. Thus the statement holds for $m=n$. The lower dimensional case follows by considering the statement in an appropriate subspace. 
Proposition 28. The functional $\ell_{1}: \mathcal{P}\left(\mathbb{Z}^{n}\right) \rightarrow \mathbb{Q}^{n}$ is an $\mathrm{SL}_{n}(\mathbb{Z})$ and translation equivariant valuation. If $P \in \mathcal{P}\left(\mathbb{Z}^{n}\right)$ is a basic simplex or centrally symmetric, then $\ell_{1}(P)=\operatorname{cen}(P)$.

Proof. That $\ell_{1}: \mathcal{P}\left(\mathbb{Z}^{n}\right) \rightarrow \mathbb{Q}^{n}$ is an $\mathrm{SL}_{n}(\mathbb{Z})$ equivariant valuation is part of Theorem 24, That $\ell_{1}$ is translation equivariant follows from Theorem 24 and (15).

If $T$ is an $m$-dimensional basic simplex, then it follows from Lemma 27 and the translation equivariance that $\ell_{1}((m+1) T)=\operatorname{cen}((m+1) T)$. As both $\ell_{1}$ and the centroid are homogeneous of degree one, we conclude $\ell_{1}(T)=\operatorname{cen}(T)$.

If $P \in \mathcal{P}\left(\mathbb{Z}^{n}\right)$ is centrally symmetric, then $\operatorname{cen}(P)$ is the center of symmetry of $P$. If $x_{0}$ is a vertex of $P$, then its image $x_{1}$ by the reflection through $\operatorname{cen}(P)$ is also a vertex. Thus $x_{0}, x_{1} \in \mathbb{Z}^{n}$ and $\operatorname{cen}(P)=\frac{1}{2}\left(x_{0}+x_{1}\right)$. The unimodular map $\phi$ defined by $z \mapsto-z+x_{0}+x_{1}$ is the reflection through $\operatorname{cen}(P)$ and its only fixed point is $\operatorname{cen}(P)$. Since both cen $(P)$ and $\ell_{1}(P)$ are fixed points of $\phi$, we conclude that $\ell_{1}(P)=\operatorname{cen}(P)$.

\subsection{Proof of Theorem 5}

That $\ell_{1}: \mathcal{P}\left(\mathbb{Z}^{n}\right) \rightarrow \mathbb{R}^{n}$ is an $\mathrm{SL}_{n}(\mathbb{Z})$ and translation equivariant valuation is part of Proposition 28. So the following proposition concludes the proof of the theorem. Let $n \geq 2$.

Proposition 29. If $\mathrm{z}: \mathcal{P}\left(\mathbb{Z}^{n}\right) \rightarrow \mathbb{R}^{n}$ is an $\mathrm{SL}_{n}(\mathbb{Z})$ and translation equivariant valuation, then $\mathrm{z}=\ell_{1}$.

Proof. Define $\mathrm{w}: \mathcal{P}\left(\mathbb{Z}^{n}\right) \rightarrow \mathbb{R}^{n}$ by $\mathrm{w}(P)=\mathrm{z}(P)-\ell_{1}(P)$. Note that $\mathrm{w}$ is an $\mathrm{SL}_{n}(\mathbb{Z})$ equivariant and translation invariant valuation. Applying Theorem 19 to w shows that if $P \in \mathcal{P}\left(\mathbb{Z}^{n}\right)$ and $k \in \mathbb{N}$, then

$$
\mathrm{w}(k P)=\sum_{i=0}^{n} \mathrm{w}_{i}(P) k^{i}
$$

where for each $i$, the function $\mathrm{w}_{i}: \mathcal{P}\left(\mathbb{Z}^{n}\right) \rightarrow \mathbb{R}^{n}$ is an $\mathrm{SL}_{n}(\mathbb{Z})$ equivariant and translation invariant valuation which is homogeneous of degree $i$.

Lemma 27 applied with $\mathrm{w}=\mathrm{w}_{i}$, the $\mathrm{SL}_{n}(\mathbb{Z})$ equivariance and translation invariance of $\mathrm{w}_{i}$ imply that

$$
\mathrm{w}_{i}\left((m+1) T_{m}\right)=\mathrm{w}_{i}\left((m+1) T_{m}-\operatorname{cen}\left((m+1) T_{m}\right)\right)=o
$$


for $i=0, \ldots, n$. Since $\mathrm{w}_{i}$ is homogeneous of degree $i$, we obtain $\mathrm{w}_{i}\left(T_{m}\right)=o$ for $i=0, \ldots, n$. Thus Corollary 18 implies that $\mathrm{w}(P)=o$ for $P \in \mathcal{P}\left(\mathbb{Z}^{n}\right)$. In particular, $\mathrm{z}(P)=\ell_{1}(P)$ for any $P \in \mathcal{P}\left(\mathbb{Z}^{n}\right)$.

\subsection{Proof of Theorem 7}

Since any additive function on $\mathcal{P}\left(\mathbb{Z}^{n}\right)$ is a valuation, Corollary 26] implies the statement of the theorem.

\section{Contravariant valuations}

In this section, we first prove Theorem 11, that is, we prove that for every $\mathrm{SL}_{2}(\mathbb{Z})$ contravariant and translation invariant Minkowski valuation $\mathrm{Z}$ on $\mathcal{P}\left(\mathbb{Z}^{2}\right)$, there are $a, b \geq 0$ such that $\mathrm{Z} P=a \rho_{\pi / 2}\left(P-\ell_{1}(P)\right)+b \rho_{\pi / 2}(-P+$ $\left.\ell_{1}(P)\right)$ for every $P \in \mathcal{P}\left(\mathbb{Z}^{2}\right)$ and we prove for $n \geq 3$ that for every $\mathrm{SL}_{n}(\mathbb{Z})$ contravariant and translation invariant Minkowski valuation $\mathrm{Z}$ on $\mathcal{P}\left(\mathbb{Z}^{n}\right)$, there is $c \geq 0$ such that $Z=c \Pi$. Second, we prove Theorem 12 ,

Note that a simple consequence of the symmetry properties of $T_{n}$ and the $\mathrm{SL}_{n}(\mathbb{Z})$ contravariance of $\mathrm{Z}$ is the following result.

Lemma 30. Let $\mathrm{Z}: \mathcal{P}\left(\mathbb{Z}^{n}\right) \rightarrow \mathcal{K}\left(\mathbb{R}^{n}\right)$ be an $\mathrm{SL}_{n}(\mathbb{Z})$ contravariant and translation invariant Minkowski valuation. If $\mathrm{ZT}_{n} \neq\{o\}$, then $o \in \operatorname{int}\left(\mathrm{ZT}_{n}\right)$.

\subsection{Lower dimensional polytopes}

The next lemma was proved in [19,28] for $\mathrm{SL}_{n}(\mathbb{R})$ equivariant (and homogeneous) valuations on $\mathcal{P}\left(\mathbb{R}^{n}\right)$.

Lemma 31. Let $\mathrm{Z}: \mathcal{P}\left(\mathbb{Z}^{n}\right) \rightarrow \mathcal{K}\left(\mathbb{R}^{n}\right)$ be an $\mathrm{SL}_{n}(\mathbb{Z})$ contravariant and translation invariant Minkowski valuation and let $P \in \mathcal{P}\left(\mathbb{Z}^{n}\right)$.

(i) If $\operatorname{dim}(P) \leq n-2$, then $\mathrm{Z} P=\{o\}$.

(ii) There exists $c \geq 0$ (depending on $\mathrm{Z}$ ) such that if $\operatorname{dim}(P)=n-1$ and $w$ is a unit normal to aff $P$, then $\mathrm{Z} P=c|P|[-w, w]$. 
Proof. By translation invariance and $\mathrm{SL}_{n}(\mathbb{Z})$ contravariance, we may assume that $\operatorname{span} P=\operatorname{span}\left\{e_{1}, \ldots, e_{d}\right\}$, where $d=\operatorname{dim}(P) \leq n-1$.

First we claim that

$$
\mathrm{Z} P \subset \operatorname{span}\left\{e_{j}\right\} \text { for } j=d+1, \ldots, n \text {. }
$$

To simplify the notation, let $j=n$ in (16).

For $j \in \mathbb{Z}$ and $k \in\{1, \ldots, n-1\}$, we define $\phi_{j k} \in \mathrm{SL}_{n}(\mathbb{Z})$ by $\phi_{j k} e_{i}=e_{i}$ if $i \neq n$, and $\phi_{j k} e_{n}=j e_{k}+e_{n}$. It follows that $\phi_{j k} P=P$. If we have $x=\sum_{i=1}^{n} t_{i} e_{i} \in \mathrm{ZP}$, then

$$
\phi_{j k}^{-t} x=\left(t_{n}-j t_{k}\right) e_{n}+\sum_{i=1}^{n-1} t_{i} e_{i}
$$

Since $\phi_{j k}^{-t} \mathrm{Z} P=\mathrm{Z} P$, the vector $\phi_{j k}^{-t} x$ is contained in a bounded set. Since $k \in\{1, \ldots, n-1\}$ and $j \in \mathbb{Z}$ are arbitrary in (17), we conclude that $t_{1}=$ $\cdots=t_{n-1}=0$. Thus (16) and therefore also (i) are proved.

To prove (ii), we identify $\operatorname{span}\left\{e_{1}, \ldots, e_{n-1}\right\}$ with $\mathbb{R}^{n-1}$. By (16), there exist real $\mathrm{z}_{1}(P) \leq \mathrm{z}_{2}(P)$ such that $\mathrm{Z} P=\left[\mathrm{z}_{1}(P), \mathrm{z}_{2}(P)\right] e_{n}$ for a lattice polytope $P \in \mathcal{P}\left(\mathbb{Z}^{n-1}\right)$. In particular, $\mathrm{z}_{1}$ and $\mathrm{z}_{2}$ are $\mathrm{SL}_{n-1}(\mathbb{Z})$ and translation invariant valuations. Let $a_{i}=\mathrm{z}_{i}\left(T_{n-1}\right) /\left|T_{n-1}\right|$. Since $\mathrm{z}_{1}(S)=\mathrm{z}_{2}(S)=0$ by (i) if $S$ is a basic simplex of dimension at most $(n-2)$, the $(n-1)$-dimensional version of Corollary 18 implies that $\mathrm{z}_{i}(P)=a_{i}|P|$ for $P \in \mathcal{P}\left(\mathbb{Z}^{n-1}\right)$.

To relate $a_{1}$ and $a_{2}$ for $n \geq 3$, we consider $\phi \in \mathrm{SL}_{n}(\mathbb{Z})$ defined by $\phi e_{1}=e_{2}$, $\phi e_{2}=e_{1}, \phi e_{n}=-e_{n}$ and $\phi e_{i}=e_{i}$ if $2<i<n$. Then $\phi T_{n-1}=T_{n-1}$ and $\phi^{-t}=\phi$. Hence $c=a_{2}=-a_{1} \geq 0$. If $n=2$, then the $\mathrm{SL}_{2}(\mathbb{Z})$ and translation invariance imply for $\psi \in \mathrm{SL}_{2}(\mathbb{Z})$ defined by $\psi e_{1}=-e_{1}$ and $\psi e_{2}=-e_{2}$ that

$$
\mathrm{Z} T_{1}=\mathrm{Z}\left(T_{1}-e_{1}\right)=\mathrm{Z}\left(\psi T_{1}\right)=\psi \mathrm{Z} T_{1} .
$$

Thus again $c=a_{2}=-a_{1} \geq 0$.

Combining Lemma 31 and the inclusion-exclusion property leads to the following result.

Corollary 32. Let $\mathrm{Z}: \mathcal{P}\left(\mathbb{Z}^{n}\right) \rightarrow \mathcal{K}\left(\mathbb{R}^{n}\right)$ be an $\mathrm{SL}_{n}(\mathbb{Z})$ contravariant and translation invariant Minkowski valuation. If $P_{1}, \ldots, P_{k} \in \mathcal{P}\left(\mathbb{Z}^{n}\right)$ form a cell decomposition of an $n$-dimensional lattice polytope, then

$$
\mathrm{Z}\left(P_{1} \cup \cdots \cup P_{k}\right)+\sum_{\operatorname{dim}\left(P_{i} \cap P_{j}\right)=n-1} \mathrm{Z}\left(P_{i} \cap P_{j}\right)=\sum_{i=1}^{k} \mathrm{Z} P_{i} .
$$




\subsection{Simple valuations}

A valuation $\mathrm{Z}$ on $\mathcal{P}\left(\mathbb{Z}^{n}\right)$ is called simple, if $\mathrm{Z} P=\{o\}$ for every lower dimensional $P \in \mathcal{P}\left(\mathbb{Z}^{n}\right)$.

Lemma 33. Let $\mathrm{Z}: \mathcal{P}\left(\mathbb{Z}^{n}\right) \rightarrow \mathcal{K}\left(\mathbb{R}^{n}\right)$ be an $\mathrm{SL}_{n}(\mathbb{Z})$ contravariant and translation invariant valuation. If $\mathrm{Z}$ is simple, then $\mathrm{Z}[0,1]^{n}=\{o\}$.

Proof. First, we consider the case $n=2$ to show the idea. In this case $[0,1]^{2}$ can be triangulated into $T_{2}$ and $T_{2}^{\prime}=e_{1}+e_{2}-T_{2}$, and hence

$$
\mathrm{Z}[0,1]^{2}=\mathrm{Z} T_{2}+\mathrm{Z} T_{2}^{\prime}
$$

The $\mathrm{SL}_{2}(\mathbb{Z})$ contravariance and the translation invariance of $\mathrm{Z}$ imply that

$$
\mathrm{Z} T_{2}^{\prime}=-\mathrm{Z} T_{2}
$$

Let $\phi \in \mathrm{SL}_{2}(\mathbb{Z})$ be defined by $\phi e_{1}=e_{2}-e_{1}, \phi e_{2}=-e_{1}$. We have $\phi T_{2}=T_{2}-e_{1}$ and $\phi T_{2}^{\prime}=T_{2}^{\prime}-2 e_{1}$. Hence $\mathrm{Z}[0,1]^{2}$ is invariant under $\phi^{-t}$. In addition, define $\psi \in \mathrm{SL}_{2}(\mathbb{Z})$ by $\psi e_{1}=-e_{2}$ and $\psi e_{2}=e_{1}$. Note that $\mathrm{Z}[0,1]^{2}$ is invariant under $\psi=\psi^{-t}$.

Suppose that there exists $x=\left(x_{1}, x_{2}\right) \in \mathrm{Z}[0,1]^{2} \backslash\{o\}$ and seek a contradiction. By the $\psi$ invariance of $\mathrm{Z}[0,1]^{2}$, we may assume that $x_{2} \neq 0$. We observe that for $\vartheta=\psi \circ \phi^{-t}$, we have

$$
\vartheta x=\left(x_{1}-x_{2}, x_{2}\right) \in \mathrm{Z}[0,1]^{2} .
$$

Since $\mathrm{Z}[0,1]^{2}$ is invariant under $\vartheta^{k}$ for any $k \geq 1$, it follows that the points $\left(x_{1}-k x_{2}, x_{2}\right) \in \mathrm{Z}[0,1]^{2}$ for any $k \geq 1$. This contradicts the boundedness of $\mathrm{Z}[0,1]^{2}$.

Next, let $n \geq 3$ and let

$$
Q=T_{2}+\sum_{i=3}^{n}\left[o, e_{i}\right] \text { and } Q^{\prime}=T_{2}^{\prime}+\sum_{i=3}^{n}\left[o, e_{i}\right]
$$

We define $\eta \in \mathrm{SL}_{n}(\mathbb{Z})$ by $\eta e_{1}=-e_{1}, \eta e_{2}=-e_{2}$ and $\eta e_{j}=e_{j}$ for $j=3, \ldots, n$.

This map satisfies $Q^{\prime}=e_{1}+e_{2}+\eta Q$ and $\eta^{-t}=\eta$. Hence

$$
\mathrm{Z} Q^{\prime}=\eta \mathrm{Z} Q
$$


In addition, let $\gamma \in \mathrm{SL}_{n}(\mathbb{Z})$ be defined by $\gamma e_{1}=e_{2}-e_{1}, \gamma e_{2}=-e_{1}$, and $\gamma e_{j}=e_{j}$ for $j=3, \ldots, n$. Thus

$$
\gamma^{-t}\left(x_{1}, x_{2}, \ldots, x_{n}\right)=\left(-x_{2}, x_{1}-x_{2}, x_{3}, \ldots, x_{n}\right) .
$$

Since $\gamma Q=Q-e_{1}$ and $\gamma$ commutes with $\eta$, it follows from (18) that

$$
\gamma^{-t} \mathrm{Z} Q=\mathrm{Z} Q \text { and } \gamma^{-t} \mathrm{Z} Q^{\prime}=\mathrm{Z} Q^{\prime}
$$

We observe that $Q$ and $Q^{\prime}$ form a polytopal cell decomposition of $[0,1]^{n}$, and hence $\mathrm{Z}[0,1]^{n}=\mathrm{Z} Q+\mathrm{Z} Q^{\prime}$. We conclude from (20) that

$$
\gamma^{-t} \mathrm{Z}[0,1]^{n}=\mathrm{Z}[0,1]^{n}
$$

Finally, suppose that there exists $x=\left(x_{1}, \ldots, x_{n}\right) \in \mathrm{Z}[0,1]^{n} \backslash\{o\}$, and seek a contradiction. For $i, m \in\{1, \ldots, n\}$ with $i \neq m$, define $\psi_{i m} \in \operatorname{SL}_{n}(\mathbb{Z})$ by setting $\psi_{i m} e_{i}=e_{m}, \psi_{i m} e_{m}=-e_{i}$ and $\psi_{i m} e_{j}=e_{j}$ for $j \neq i, m$. By the $\psi_{i, i+1}$ invariance of $[0,1]^{n}$ for $i=1, \ldots, n-1$, we may assume that $x_{2} \neq 0$. We deduce by (19) that $\vartheta=\psi_{21} \circ \gamma^{-t}$ satisfies

$$
\vartheta x=\left(x_{1}-x_{2}, x_{2}, x_{3}, \ldots, x_{n}\right) \in \mathrm{Z}[0,1]^{n} .
$$

Since $\mathrm{Z}[0,1]^{n}$ is invariant under $\vartheta^{k}$ for any $k \geq 1$, it follows that

$$
\left(x_{1}-k x_{2}, x_{2}, x_{3}, \ldots, x_{n}\right) \in \mathrm{Z}[0,1]^{n}
$$

for any $k \geq 1$. This contradicts the boundedness of $\mathrm{Z}[0,1]^{n}$.

\subsection{The cube}

Let $n \geq 2$ and recall that the constant $c$ was defined in Lemma 31 .

Lemma 34. If $\mathrm{Z}: \mathcal{P}\left(\mathbb{Z}^{n}\right) \rightarrow \mathcal{K}\left(\mathbb{R}^{n}\right)$ is an $\mathrm{SL}_{n}(\mathbb{Z})$ contravariant and translation invariant Minkowski valuation, then

$$
\mathrm{Z}\left(k[0,1]^{n}\right)+c\left(k^{n}-k^{n-1}\right) \Pi[0,1]^{n}=k^{n} \mathrm{Z}[0,1]^{n}
$$

for $k \in \mathbb{N}$. 
Proof. For $k \geq 1$, we subdivide $k[0,1]^{n}$ into a cell decomposition of $k^{n}$ unit cubes, and hence all $m$-dimensional faces are unit cubes of dimension $m$. For $i=1, \ldots, n$, there exist $k^{n-1}(k-1)$ facets of the cell decomposition which intersect the interior of $k[0,1]^{n}$ and whose unit normal vector is $e_{i}$. We deduce from Lemma 31 and Corollary 32 that

$$
\mathrm{Z}\left(k[0,1]^{n}\right)+\left(k^{n}-k^{n-1}\right) \sum_{i=1}^{n} c\left[-e_{i}, e_{i}\right]=k^{n} \mathrm{Z}[0,1]^{n} .
$$

The definition of the projection body, (3) , gives $[-c, c]^{n}=c \Pi[0,1]^{n}$.

Proposition 35. If $\mathrm{Z}: \mathcal{P}\left(\mathbb{Z}^{n}\right) \rightarrow \mathcal{K}\left(\mathbb{R}^{n}\right)$ is an $\mathrm{SL}_{n}(\mathbb{Z})$ contravariant and translation invariant Minkowski valuation, then $\mathrm{Z}[0,1]^{n}=c \Pi[0,1]^{n}$.

Proof. Consider the $\mathrm{SL}_{n}(\mathbb{Z})$ contravariant and translation invariant valuation $\mathrm{Z}_{n}$ defined in Lemma 20. Since $\mathrm{Z}_{n}$ is homogeneous of degree $n$, we deduce from Lemma 34 applied to $\mathrm{Z}_{n}$ that $\mathrm{Z}_{n}$ is simple. Lemma 33 implies that $\mathrm{Z}_{n}[0,1]^{n}=\{o\}$. In particular, we have $\lim _{k \rightarrow \infty} \mathrm{Z}\left(k[0,1]^{n}\right) / k^{n}=\{o\}$. Next we apply Lemma 34 to Z. Dividing both sides by $k^{n}$ and letting $k \rightarrow \infty$ implies that $\mathrm{Z}[0,1]^{n}=c \Pi[0,1]^{n}$.

\subsection{The planar case}

It is easy to see that

$$
\rho_{\pi / 2} \phi \rho_{-\pi / 2}=\phi^{-t} \text { for any } \phi \in \mathrm{SL}_{2}(\mathbb{R}) \text {. }
$$

As in [30], we deduce the following result.

Lemma 36. An operator $\mathrm{Z}: \mathcal{P}\left(\mathbb{Z}^{2}\right) \rightarrow \mathcal{K}\left(\mathbb{R}^{2}\right)$ is $\mathrm{SL}_{2}(\mathbb{Z})$ equivariant if and only if $\rho_{\pi / 2} \mathrm{Z}: \mathcal{P}\left(\mathbb{Z}^{2}\right) \rightarrow \mathcal{K}\left(\mathbb{R}^{2}\right)$ is $\mathrm{SL}_{2}(\mathbb{Z})$ contravariant.

For the next lemma, recall that for given $\mathrm{Z}$, the constant $c$ was defined in Lemma 31 .

Lemma 37. If $\mathrm{Z}: \mathcal{P}\left(\mathbb{Z}^{2}\right) \rightarrow \mathcal{K}\left(\mathbb{R}^{2}\right)$ is an $\mathrm{SL}_{2}(\mathbb{Z})$ contravariant and translation invariant Minkowski valuation, then there exist $a, b \geq 0$ such that

$$
\mathrm{Z} T_{2}=a \rho_{\pi / 2}\left(T_{2}-\operatorname{cen}\left(T_{2}\right)\right)+b \rho_{\pi / 2}\left(-T_{2}+\operatorname{cen}\left(T_{2}\right)\right)
$$

and $a+b=2 c$. 
Proof. We dissect $[0,1]^{2}$ into the triangles $T_{2}$ and $T_{2}^{\prime}=e_{1}+e_{2}-T_{2}$. Since Z is a valuation,

$$
\mathrm{Z}[0,1]^{2}+\mathrm{Z}\left(T_{2} \cap T_{2}^{\prime}\right)=\mathrm{Z} T_{2}+\mathrm{Z} T_{2}^{\prime} .
$$

Combining Lemma 31 and Proposition 35 leads to

$$
c[-1,1]^{2}+c\left[-\left(e_{1}+e_{2}\right), e_{1}+e_{2}\right]=\mathrm{Z} T_{2}+\mathrm{Z} T_{2}^{\prime} .
$$

Therefore $\mathrm{Z} T_{2}$ is a Minkowski summand of the hexagon on the left hand side. If $c=0$, then $\mathrm{ZT}_{2}=\{o\}$, and Lemma 31 implies that Z is simple. Therefore $\mathrm{Z} P=\{o\}$ for all $P \in \mathcal{P}^{2}$. So let $c>0$. Since $\mathrm{Z}$ is $\mathrm{SL}_{2}(\mathbb{Z})$ contravariant, we have $\mathrm{Z} T_{2}^{\prime}=-\mathrm{Z} T_{2}$ and (21) implies that $\mathrm{Z} T_{2}$ is a two-dimensional polygon whose sides are parallel to $e_{1}$ or $e_{2}$ or $e_{1}+e_{2}$. In addition, let $\phi \in \mathrm{SL}_{2}(\mathbb{Z})$ be defined by $\phi e_{1}=e_{2}-e_{1}$ and $\phi e_{2}=-e_{1}$. Then $\mathrm{ZT}_{2}$ is invariant under $\phi^{-t}$. Since $\phi$ permutes $e_{1},-e_{1}+e_{2},-e_{2}$ on the one hand and $-e_{1}, e_{1}-e_{2}, e_{2}$ on the other hand, we have $h\left(\mathrm{Z} T_{2}, e_{1}\right)=h\left(\mathrm{Z} T_{2},-e_{1}+e_{2}\right)=h\left(\mathrm{Z} T_{2},-e_{2}\right)$ and $h\left(\mathrm{ZT}_{2},-e_{1}\right)=h\left(\mathrm{ZT} T_{2}, e_{1}-e_{2}\right)=h\left(\mathrm{ZT}_{2}, e_{2}\right)$. Thus it is easy to check that

$$
\mathrm{Z} T_{2}=a \rho_{\pi / 2}\left(T_{2}-\operatorname{cen}\left(T_{2}\right)\right)+b \rho_{\pi / 2}\left(-T_{2}+\operatorname{cen}\left(T_{2}\right)\right)
$$

for suitable $a, b \geq 0$. From (21) we obtain $a+b=2 c$.

Proof of Theorem 11 in the planar case. It follows from Proposition 28 that $P \mapsto P-\ell_{1}(P)$ and $P \mapsto-P+\ell_{1}(P)$ are $\mathrm{SL}_{2}(\mathbb{Z})$ equivariant and translation invariant Minkowski valuations on $\mathcal{P}\left(\mathbb{Z}^{2}\right)$. We deduce from Lemma 36 that $P \mapsto \rho_{\pi / 2}\left(P-\ell_{1}(P)\right)$ and $P \mapsto \rho_{\pi / 2}\left(-P+\ell_{1}(P)\right)$ are $\mathrm{SL}_{2}(\mathbb{Z})$ contravariant and translation invariant Minkowski valuations.

Since $\ell_{1}\left(T_{i}\right)=\operatorname{cen}\left(T_{i}\right)$ for $i=1,2$ by Proposition 28, combining Lemma 37, Lemma 31 and Corollary 18 shows that any $\mathrm{SL}_{2}(\mathbb{Z})$ contravariant and translation invariant Minkowski valuation $\mathrm{Z}$ is of the form

$$
\mathrm{Z} P=a \rho_{\pi / 2}\left(P-\ell_{1}(P)\right)+b \rho_{\pi / 2}\left(-P+\ell_{1}(P)\right)
$$

where $a, b \geq 0$.

\subsection{Proof of Theorem 11 for $n \geq 3$}

For Minkowski summands, we need the following (probably well-known) statement, for which we have not found a reference. Let $n \geq 3$. 
Lemma 38. Let $v_{1}, \ldots, v_{m}$ be vectors in $\mathbb{R}^{n}$ such that any $n$ of these vectors are linearly independent. If $P$ is an $n$-dimensional polytope such that every edge of $P$ is parallel to some $v_{i}$, then $P$ is a translate of $\sum_{i=1}^{m} a_{i}\left[o, v_{i}\right]$ with $a_{i} \geq 0$.

Proof. A polytope is a zonotope, if all its two-dimensional faces are centrally symmetric (cf. [48, Theorem 3.5.2]). Thus it is sufficient to show that $P$ has centrally symmetric two-dimensional faces.

We may assume that $P$ has an edge parallel to $v_{i}$ for every $i=1, \ldots, m$. Let $[x, y]$ be an edge of $P$ parallel to $v_{m}$ where $y=x+a_{m} v_{m}$ for $a_{m}>0$. We claim that for any vertex $w$ of $\pi_{v_{m}} P$, there exists a vertex $z$ of $P$ such that

$$
\pi_{v_{m}} z=w \text { and } z+a_{m} v_{m} \text { is a vertex of } P \text {. }
$$

Since there is a path of the edge graph of $\pi_{v_{m}} P$ connecting $\pi_{v_{m}} x$ and $w$, we may assume that $\left[w, \pi_{v_{m}} x\right]$ is an edge of $\pi_{v_{m}} P$. Let $L$ be the span of $v_{m}$ and $w-\pi_{v_{m}} x$. It follows that $(x+L) \cap P$ is a two-dimensional face of $P$. As no three vectors from $V=\left\{v_{1}, \ldots, v_{m}\right\}$ are linearly dependent, we deduce that for some $i \in\{1, \ldots, m-1\}$ we have $L \cap V=\left\{v_{i}, v_{m}\right\}$. As the twodimensional face $F=(x+L) \cap P$ has only edges parallel to $v_{i}$ and $v_{m}$, it is a parallelogram and hence centrally-symmetric.

Let $c \geq 0$ be the constant of Lemma 31. First we use the triangulation $S_{1}, \ldots, S_{n !}$ of $[0,1]^{n}$ into basic simplices given by Lemma 16 with $S_{1}=T_{n}$. If $\operatorname{dim}\left(S_{i} \cap S_{j}\right)=n-1$, then Lemma 31 provides a non-zero $p_{i j} \in \mathbb{R}^{n}$ such that $\mathrm{Z}\left(S_{i} \cap S_{j}\right)=c\left[-p_{i j}, p_{i j}\right]$. Applying Corollary 32 and Proposition 35 to the cell decomposition of $[0,1]^{n}$, we deduce that

$$
[-c, c]^{n}+\sum_{\operatorname{dim}\left(S_{i} \cap S_{j}\right)=n-1} c\left[-p_{i j}, p_{i j}\right]=\sum_{i=1}^{n !} \mathrm{Z} S_{i} .
$$

If $c=0$, then (23) implies that $\mathrm{ZT}_{n}=\mathrm{Z} S_{1}=\{o\}$ and we conclude from Lemma 31 and Corollary 18 that $\mathrm{Z} P=\{o\}$ for $P \in \mathcal{P}\left(\mathbb{Z}^{n}\right)$.

Assume that $c>0$. Hence the left hand side of (23) is full dimensional. Since Z $S_{i}=\phi_{i}^{-t} \mathrm{Z} T_{n}$ for $\phi_{i} \in \mathrm{SL}_{n}(\mathbb{Z})$ with $S_{i}=\phi_{i} T_{n}$, it follows that $\mathrm{Z} T_{n} \neq$ $\{o\}$. We deduce from Lemma 30 that $\operatorname{dim}\left(Z T_{n}\right)=n$.

We consider the decomposition of $[0,1]^{n}$ into $T_{n}$ and $R_{n}$ from (6). For $w=-e_{1}-\cdots-e_{n}$, Lemma 31 implies that

$$
\mathrm{Z}\left[e_{1}, \ldots, e_{n}\right]=\frac{c}{(n-1) !}[-w, w] .
$$


Therefore it follows from Proposition 35 that (6) can be written in the form

$$
[-c, c]^{n}+\frac{c}{(n-1) !}[-w, w]=\mathrm{Z} T_{n}+\mathrm{Z} R_{n} .
$$

We deduce from (24) right away that $\mathrm{ZT}_{n}$ is a polytope. Each edge of the left side of (24) is parallel to either $w$ or to an $e_{i}, i=1, \ldots, n$. As any $n$ of the vectors $w, e_{1}, \ldots, e_{n}$ are linearly independent, (24) and Lemma 38 imply that

$$
\mathrm{Z} T_{n} \text { is a translate of } a_{0}[o, w]+a_{1}\left[o, e_{1}\right]+\cdots+a_{n}\left[o, e_{n}\right]
$$

where $a_{i} \geq 0$ for $i=0, \ldots, n$.

Let $\alpha$ be defined as in (9) and let $\rho \in \operatorname{Sym}\left(T_{*}\right)$. Note that the map $\alpha^{-1} \rho \alpha \in \mathrm{SL}_{n}(\mathbb{Z})$. Hence, by the $\mathrm{SL}_{n}(\mathbb{R})$ contravariance of $\mathrm{Z}$,

$$
\alpha^{-t} \mathrm{Z} T_{n}=\rho \alpha^{-t} \mathrm{Z} T_{n} .
$$

Since this holds for all $\rho \in \operatorname{Sym}\left(T_{*}\right)$, it follows that $o=\operatorname{cen}\left(\alpha^{-t} \mathrm{Z} T_{n}\right)=$ $\operatorname{cen}\left(\mathrm{Z} T_{n}\right)$. Since $\rho \in \operatorname{Sym}\left(T_{*}\right)$ permutes the normal vectors of $T_{*}$, it permutes $\alpha^{-t} w, \alpha^{-t} e_{1}, \ldots, \alpha^{-t} e_{n}$ and we obtain $a_{0}=\cdots=a_{n}$ in (25). Taking into account (3), we conclude that $\mathrm{Z} T_{n}=c_{0} \Pi T_{n}$ for $c_{0}>0$.

To determine $c_{0}$, we deduce from (23) that

$$
[-c, c]^{n}+\sum_{\operatorname{dim}\left(S_{i} \cap S_{j}\right)=n-1} c\left[-p_{i j}, p_{i j}\right]=c_{0} \sum_{i=1}^{n !} \phi_{i}^{-t} \Pi T_{n} .
$$

The $\mathrm{SL}_{n}(\mathbb{Z})$ contravariant and translation invariant Minkowski valuation $c \Pi$ also satisfies (23). Hence $c_{0}=c$. Thus Theorem 11 follows from Corollary 18 .

\subsection{Proof of Theorem 12}

First, let $n=2$. Propositions $\left[17\right.$ and 28 imply that $6 \ell_{1}(P) \in \mathbb{Z}^{2}$ for all $P \in \mathcal{P}\left(\mathbb{Z}^{2}\right)$. Hence, for integers $a, b \geq 0$ with $b-a \in 6 \mathbb{Z}$, the operator $\mathrm{Z}$ defined by

$$
P \mapsto a \rho_{\pi / 2}\left(P-\ell_{1}(P)\right)+b \rho_{\pi / 2}\left(-P+\ell_{1}(P)\right)
$$

maps $\mathcal{P}\left(\mathbb{Z}^{2}\right)$ to $\mathcal{P}\left(\mathbb{Z}^{2}\right)$. For the reverse direction, let $\mathrm{Z}: \mathcal{P}\left(\mathbb{Z}^{2}\right) \rightarrow \mathcal{P}\left(\mathbb{Z}^{2}\right)$ be an $\mathrm{SL}_{2}(\mathbb{Z})$ contravariant and translation invariant Minkowski valuation. By Theorem 11, we know that there are $a, b \geq 0$ such that

$$
\mathrm{Z} P=a \rho_{\pi / 2}\left(P-\ell_{1}(P)\right)+b \rho_{\pi / 2}\left(-P+\ell_{1}(P)\right)
$$


for every $P \in \mathcal{P}\left(\mathbb{Z}^{2}\right)$. Since $\mathrm{Z} P \in \mathcal{P}\left(\mathbb{Z}^{2}\right)$ for all $P \in \mathcal{P}\left(\mathbb{Z}^{2}\right)$, setting $P=T_{1}$ shows that $a+b \in 2 \mathbb{Z}$ and setting $P=T_{2}$ shows that $2 a+b, a+2 b \in 3 \mathbb{Z}$. Thus $a, b \in \mathbb{Z}$ and $b-a \in 6 \mathbb{Z}$.

Next, let $n \geq 3$. By (3) and (4) , the projection body of $P \in \mathcal{P}\left(\mathbb{Z}^{n}\right)$ with facet normals $u_{1}, \ldots, u_{m}$ and corresponding facets $F_{1}, \ldots, F_{m}$ is

$$
\sum_{i=1}^{m}\left|F_{i}\right|\left[o, u_{i}\right] .
$$

Since every facet can be triangulated and the $(n-1)$-dimensional volume of an $(n-1)$-dimensional lattice simplex is an integer multiple of $1 /(n-1)$ !, we have $P \mapsto c \Pi P$ with $c \in(n-1) ! \mathbb{Z}$ is an operator that maps $\mathcal{P}\left(\mathbb{Z}^{n}\right)$ to $\mathcal{P}\left(\mathbb{Z}^{n}\right)$. For the reverse direction, let $\mathrm{Z}: \mathcal{P}\left(\mathbb{Z}^{n}\right) \rightarrow \mathcal{P}\left(\mathbb{Z}^{n}\right)$ be an $\mathrm{SL}_{n}(\mathbb{Z})$ contravariant and translation invariant Minkowski valuation. By Theorem 11, we know that there is $c \geq 0$ such that $\mathrm{Z} P=c \Pi P$ for every $P \in \mathcal{P}\left(\mathbb{Z}^{n}\right)$. Since $\Pi T_{n-1}=1 /(n-1) !\left[-e_{n}, e_{n}\right]$, we conclude that $c \in(n-1) ! \mathbb{Z}$.

\section{Equivariant valuations}

For $a, b \geq 0$, define $\mathrm{Z}_{a, b}: \mathcal{P}\left(\mathbb{Z}^{n}\right) \rightarrow \mathcal{K}\left(\mathbb{R}^{n}\right)$ by

$$
\mathrm{Z}_{a, b} P=a\left(P-\ell_{1}(P)\right)+b\left(-P+\ell_{1}(P)\right) .
$$

Note that $\mathrm{Z}_{a, b}$ is an $\mathrm{SL}_{n}(\mathbb{Z})$ equivariant and translation invariant Minkowski valuation on $\mathcal{P}\left(\mathbb{Z}^{n}\right)$. In this section, we prove Theorem 4 , that is, we prove that for every $\mathrm{SL}_{n}(\mathbb{Z})$ equivariant and translation invariant Minkowski valuation $\mathrm{Z}$ on $\mathcal{P}\left(\mathbb{Z}^{n}\right)$, there are $a, b \geq 0$ such that $\mathrm{Z}=\mathrm{Z}_{a, b}$.

We deduce from Theorem 11 in the planar case and from Lemma 36 the planar case of Theorem 4 .

Proposition 39. If $\mathrm{Z}: \mathcal{P}\left(\mathbb{Z}^{2}\right) \rightarrow \mathcal{K}\left(\mathbb{R}^{2}\right)$ is an $\mathrm{SL}_{2}(\mathbb{Z})$ equivariant and translation invariant Minkowski valuation, then there exist $a, b \geq 0$ such that $\mathrm{Z}=\mathrm{Z}_{a, b}$.

As in the contravariant case, the following lemma is a simple consequence of the symmetry properties of $T_{n}$ and the equivariance of $\mathrm{Z}$.

Lemma 40. Let $\mathrm{Z}: \mathcal{P}\left(\mathbb{Z}^{n}\right) \rightarrow \mathcal{K}\left(\mathbb{R}^{n}\right)$ be an $\mathrm{SL}_{n}(\mathbb{Z})$ equivariant and translation invariant Minkowski valuation. If $\mathrm{Z} T_{n} \neq\{o\}$, then $o \in \operatorname{int}\left(\mathrm{ZT}_{n}\right)$. 
The proof of the following result is analogous to the proof of Lemma 33 in the contravariant case.

Lemma 41. Let $\mathrm{Z}: \mathcal{P}\left(\mathbb{Z}^{n}\right) \rightarrow \mathcal{K}\left(\mathbb{R}^{n}\right)$ be an $\mathrm{SL}_{n}(\mathbb{Z})$ equivariant and translation invariant valuation. If $\mathrm{Z}$ is simple, then $\mathrm{Z}[0,1]^{n}=\{o\}$.

\subsection{Lower dimensional polytopes}

In this section, we derive results on the image under a Minkowski valuation of lower dimensional lattice polytopes. The next lemma was proved in [19,30] for $\mathrm{SL}_{n}(\mathbb{R})$ equivariant (and homogeneous) valuations on $\mathcal{P}\left(\mathbb{R}^{n}\right)$. Let $n \geq 2$.

Lemma 42. If $\mathrm{Z}: \mathcal{P}\left(\mathbb{Z}^{n}\right) \rightarrow \mathcal{K}\left(\mathbb{R}^{n}\right)$ is an $\mathrm{SL}_{n}(\mathbb{Z})$ equivariant and translation invariant Minkowski valuation, then $\mathrm{Z} P$ is contained in the subspace parallel to aff $P$.

Proof. By translation invariance and $\mathrm{SL}_{n}(\mathbb{Z})$ equivariance, we may assume that $\operatorname{span} P=\operatorname{span}\left\{e_{1}, \ldots, e_{d}\right\}$, where $d=\operatorname{dim}(P) \leq n-1$.

For $j \in \mathbb{Z}$ and $k \in\{d+1, \ldots, n\}$, we define $\phi_{j k} \in \mathrm{SL}_{n}(\mathbb{Z})$ by $\phi_{j k} e_{i}=e_{i}$ for $i \neq k$ and $\phi_{j k} e_{k}=e_{k}+j e_{1}$. It follows that $\phi_{j k} P=P$. If $x=\sum_{i=1}^{n} t_{i} e_{i} \in \mathrm{Z} P$, then

$$
\phi_{j k} x=\left(t_{1}+j t_{k}\right) e_{1}+\sum_{i=2}^{n} t_{i} e_{i} .
$$

Since $\phi_{j k} \mathrm{Z} P=\mathrm{Z} P$, the convex body $\mathrm{Z} P$ is bounded, and $k \in\{d+1, \ldots, n\}$ and $j \in \mathbb{Z}$ are arbitrary in (27), we conclude that $t_{d+1}=\cdots=t_{n}=0$.

\subsection{The cube}

Proposition 43 is the main result of this section. Let $n \geq 2$.

Proposition 43. If $\mathrm{Z}: \mathcal{P}\left(\mathbb{Z}^{n}\right) \rightarrow \mathcal{K}\left(\mathbb{R}^{n}\right)$ is an $\mathrm{SL}_{n}(\mathbb{Z})$ equivariant and translation invariant Minkowski valuation, then there exists $c \geq 0$ such that

$$
\mathrm{Z}[0,1]^{m}=[-c, c]^{m}
$$

for $m=0, \ldots, n$.

The critical step to prove Proposition 43 is the following statement where $[a, b]^{0}=\{o\}$ for $a \leq b$. 
Lemma 44. If $\mathrm{Z}: \mathcal{P}\left(\mathbb{Z}^{n}\right) \rightarrow \mathcal{K}\left(\mathbb{R}^{n}\right)$ is an $\mathrm{SL}_{n}(\mathbb{Z})$ equivariant and translation invariant Minkowski valuation and there exists $c \geq 0$ such that $\mathrm{Z}[0,1]^{m}=$ $[-c, c]^{m}$ for every $m \leq n-1$, then

$$
\mathrm{Z}\left(k[0,1]^{n}\right)+k^{n}[-c, c]^{n}=k^{n} \mathrm{Z}[0,1]^{n}+k[-c, c]^{n}
$$

for $k \in \mathbb{N}$.

Proof. For $k \geq 1$, we subdivide $k[0,1]^{n}$ into a cell decomposition with $k^{n}$ unit cubes. We observe that for $m=1, \ldots, n-1$,

$$
\sum_{1 \leq i_{1}<\cdots<i_{m} \leq n} \sum_{j=1}^{m}\left[-e_{i_{j}}, e_{i_{j}}\right]=\left(\begin{array}{c}
n-1 \\
m-1
\end{array}\right)[-1,1]^{n}
$$

and for $m=0, \ldots, n-1$, there exist $k^{m}(k-1)^{n-m}$ translates of $[0,1]^{m}$ that are faces of the cell decomposition intersecting the interior of $k[0,1]^{n}$. Since $\mathrm{Z}[0,1]^{m}=[-c, c]^{m}$ for $m \leq n-1$, we have $\mathrm{Z}\{o\}=\{o\}$, and we deduce from Corollary 15 that for $v \in \mathbb{R}^{n}$

$$
\begin{aligned}
& h\left(\mathrm{Z}\left(k[0,1]^{n}\right), v\right)= \\
& \quad=k^{n} h\left(\mathrm{Z}[0,1]^{n}, v\right)+\sum_{m=1}^{n-1}(-1)^{n-m}\left(\begin{array}{c}
n-1 \\
m-1
\end{array}\right) k^{m}(k-1)^{n-m} h\left([-c, c]^{n}, v\right) \\
& \quad=k^{n} h\left(\mathrm{Z}[0,1]^{n}, v\right)+k \sum_{j=0}^{n-2}\left(\begin{array}{c}
n-1 \\
j
\end{array}\right) k^{j}(1-k)^{n-1-j} h\left([-c, c]^{n}, v\right) \\
& \quad=k^{n} h\left(\mathrm{Z}[0,1]^{n}, v\right)+k\left((k+1-k)^{n-1}-k^{n-1}\right) h\left([-c, c]^{n}, v\right) \\
& \quad=k^{n} h\left(\mathrm{Z}[0,1]^{n}, v\right)-k^{n} h\left([-c, c]^{n}, v\right)+k h\left([-c, c]^{n}, v\right) .
\end{aligned}
$$

Thus the lemma is proved.

Proof of Proposition 43. We prove the statement by induction on $n \geq 2$. The case $n=2$ follows from Proposition 39 and the fact that $\ell_{1}\left([0,1]^{m}\right)$ is the centroid of $[0,1]^{m}$ by Proposition 28 .

Let $n \geq 3$ and assume that Proposition 43 holds for $m \leq n-1$. We consider the $\mathrm{SL}_{n}(\mathbb{Z})$ equivariant and translation invariant Minkowski valuation $\mathrm{Z}_{n}$ defined in Lemma 20. Since $\mathrm{Z}_{n}$ is homogeneous of degree $n$, we deduce from Lemma 44 applied to $\mathrm{Z}_{n}$ that $\mathrm{Z}_{n}[0,1]^{m}=\{o\}$ for $m \leq n-1$. Hence $\mathrm{Z}_{n}$ is simple and we obtain by Lemma 41 that

$$
\mathrm{Z}_{n}[0,1]^{n}=\lim _{k \rightarrow \infty} \frac{\mathrm{Z}\left(k[0,1]^{n}\right)}{k^{n}}=\{o\} .
$$


Next, we apply Lemma 44 to Z. Dividing both sides by $k^{n}$, and letting $k \rightarrow \infty$ shows that $\mathrm{Z}[0,1]^{n}=[-c, c]^{n}$.

\subsection{The prism}

Let $\mathrm{Z}: \mathcal{P}\left(\mathbb{Z}^{n}\right) \rightarrow \mathcal{K}\left(\mathbb{R}^{n}\right)$ be an $\mathrm{SL}_{n}(\mathbb{Z})$ equivariant and translation invariant Minkowski valuation. Let $n \geq 3$.

Lemma 45. If $S \in \mathcal{P}\left(\mathbb{Z}^{n-1}\right)$ is a basic simplex and $k \in \mathbb{N}$, then

$$
\operatorname{cen}\left(\mathrm{Z}\left(S+\left[o, k e_{n}\right]\right)\right)=o .
$$

Proof. We may assume that $S=T_{m}$ for some $m=0, \ldots, n-1$. If $m=0,1$, then $\phi\left(T_{m}+\left[0, k e_{n}\right]\right)$ is a translate of $T_{m}+\left[0, k e_{n}\right]$ where $\phi \in \mathrm{SL}_{n}(\mathbb{Z})$ is defined by $\phi e_{1}=-e_{1}, \phi e_{n}=-e_{n}$ and $\phi e_{j}=e_{j}$ for $j=2, \ldots, n-1$. Since we have $\mathrm{Z}\left(T_{m}+\left[0, k e_{n}\right]\right) \subset \operatorname{span}\left\{e_{1}, e_{n}\right\}$ by Lemma 42 , we deduce the statement of the lemma for $m=0,1$.

If $m \geq 2$, then $\mathrm{Z}\left(T_{m}+\left[0, k e_{n}\right]\right) \subset \operatorname{span}\left\{e_{1}, \ldots, e_{m}, e_{n}\right\}$ by Lemma 42. Hence we may assume that $m=n-1$. Let $\alpha^{\prime} \in \mathrm{GL}_{n}(\mathbb{R})$ be the transformation that leaves $e_{n}$ fixed and acts on $\mathbb{R}^{n-1}$ as $\alpha$ defined in Section 2.8. Then $\alpha^{\prime} \mathrm{Z}\left(T_{n-1}+\left[o, k e_{n}\right]\right)$ is invariant under the maps $\rho^{\prime}$ that leave $e_{n}$ fixed and are orientation preserving isometries of the regular simplex $\left[v_{0}, \ldots, v_{n-1}\right] \subset \mathbb{R}^{n-1}$ defined in Section 2.8. Thus the first $(n-1)$ coordinates of the centroid of $\alpha^{\prime} \mathrm{Z}\left(T_{n-1}+\left[o, k e_{n}\right]\right)$ vanish. In addition, $\mathrm{Z}\left(T_{n-1}+\left[o, k e_{n}\right]\right)$ is invariant under the map $\psi \in \mathrm{SL}_{n}(\mathbb{Z})$ defined by $\psi e_{n}=-e_{n}, \psi e_{1}=e_{2}, \psi e_{2}=e_{1}$, and $\psi e_{j}=e_{j}$ for $2<j<n$. This completes the proof of the lemma.

Recall that $\widetilde{T}_{n-1}=T_{n-1}+\left[0, e_{n}\right]$.

Lemma 46. Assume that Theorem 4 holds true in dimension $(n-1)$ and hence that there exist $a, b \geq 0$ such that $\mathrm{Z} P=\mathrm{Z}_{a, b} P$ for every lower dimensional $P \in \mathcal{P}\left(\mathbb{Z}^{n}\right)$. Then

$$
\mathrm{Z} \widetilde{T}_{n-1}=\mathrm{Z}_{a, b} \widetilde{T}_{n-1}
$$

Proof. We define the convex body $\mathrm{Z}^{\prime} P \subset \mathbb{R}^{n-1}$ for $P \in \mathcal{P}\left(\mathbb{Z}^{n-1}\right)$ by

$$
\mathrm{Z}^{\prime} P=\pi_{e_{n}} \mathrm{Z}\left(P+\left[o, e_{n}\right]\right)
$$


Then $\mathrm{Z}^{\prime}: \mathcal{P}\left(\mathbb{Z}^{n-1}\right) \rightarrow \mathcal{K}\left(\mathbb{R}^{n-1}\right)$ is an $\mathrm{SL}_{n-1}(\mathbb{Z})$ equivariant and translation invariant Minkowski valuation. Since Theorem 4 holds in dimension $(n-1)$, there exist $a^{\prime}, b^{\prime} \geq 0$ such that

$$
\mathrm{Z}^{\prime} P=\mathrm{Z}_{a^{\prime}, b^{\prime}} P \text { for } P \in \mathcal{P}\left(\mathbb{Z}^{n-1}\right) .
$$

By Proposition 43, we have

$$
[-c, c]^{n-1}=\mathrm{Z}[0,1]^{n-1}=\mathrm{Z}^{\prime}[0,1]^{n-1}=\mathrm{Z}_{a^{\prime}, b^{\prime}}[0,1]^{n-1} .
$$

Combined with the assumption that $\mathrm{Z} P=\mathrm{Z}_{a, b} P$ for every lower dimensional $P \in \mathcal{P}\left(\mathbb{Z}^{n}\right)$ and Proposition 28 this gives

$$
a^{\prime}+b^{\prime}=2 c=a+b .
$$

For $m=(n-1)$ !, we consider the triangulation $S_{1}^{\prime}, \ldots, S_{m}^{\prime}$ of $[0,1]^{n-1}$ into $(n-1)$-dimensional basic simplices with $S_{1}^{\prime}=T_{n-1}$ provided by Lemma 16. For $i=1, \ldots, m$, set $\widetilde{S}_{i}^{\prime}=S_{i}^{\prime}+\left[o, e_{n}\right]$. Note that the prisms $\widetilde{S}_{1}^{\prime}, \ldots, \widetilde{S}_{m}^{\prime}$ form a cell decomposition of $[0,1]^{n}$. Let $\mathcal{F}$ denote the family of faces of the cell decomposition intersecting the interior of $[0,1]^{n}$. It follows from the inclusion-exclusion principle that

$$
\mathrm{Z}[0,1]^{n}+\sum_{\substack{F \in \mathcal{F} \\ n-\operatorname{dim}(F) \text { odd }}} \mathrm{ZF}=\sum_{\substack{F \in \mathcal{F} \\ n-\operatorname{dim}(F) \text { even }}} \mathrm{ZF} .
$$

We relate $\mathrm{Z}$ to $\mathrm{Z}_{a, b}$. Note that $\mathrm{Z}_{a, b}$ in place of $\mathrm{Z}$ also satisfies (30). Since $\mathrm{Z}[0,1]^{n}=\mathrm{Z}_{a, b}[0,1]^{n}$ by Proposition 43 and (29), and $\mathrm{Z} F=\mathrm{Z}_{a, b} F$ for lower dimensional lattice polytopes $F \in \mathcal{P}\left(\mathbb{Z}^{n}\right)$, we deduce that

$$
\sum_{i=1}^{m} \mathrm{Z} \widetilde{S}_{i}^{\prime}=\sum_{i=1}^{m} \mathrm{Z}_{a, b} \widetilde{S}_{i}^{\prime}
$$

For $i=1, \ldots, m$, we have

$$
\mathrm{Z}_{a, b} \widetilde{S}_{i}^{\prime}=a\left(S_{i}^{\prime}-\operatorname{cen}\left(S_{i}^{\prime}\right)\right)+b\left(-S_{i}^{\prime}+\operatorname{cen}\left(S_{i}^{\prime}\right)\right)+c\left[-e_{n}, e_{n}\right]
$$

by the Minkowski linearity of $\ell_{1}$, Proposition 28 and (29). Hence the right hand side of (31) is of the form $Q+c m\left[-e_{n}, e_{n}\right]$ for a suitable $(n-1)$ dimensional polytope $Q \subset \mathbb{R}^{n-1}$. It follows from (31) that $\mathrm{Z} \widetilde{T}_{n-1}=\mathrm{Z} \widetilde{S}_{1}^{\prime}$ is an $n$-dimensional polytope that is a summand of $Q+c m\left[-e_{n}, e_{n}\right]$. Hence, 
because of (2), the facet outer normals of $\mathrm{Z} \widetilde{T}_{n-1}$ are either parallel or orthogonal to $e_{n}$. Hence, by Lemma 45, there exists $c_{0}>0$ such that

$$
\mathrm{Z} \widetilde{T}_{n-1}=\mathrm{Z}^{\prime} T_{n-1}+c_{0}\left[-e_{n}, e_{n}\right] .
$$

By (28) and Proposition 28, we therefore get

$$
\mathrm{Z} \widetilde{T}_{n-1}=a^{\prime}\left(T_{n-1}-\operatorname{cen}\left(T_{n-1}\right)\right)+b^{\prime}\left(-T_{n-1}+\operatorname{cen}\left(T_{n-1}\right)\right)+c_{0}\left[-e_{n}, e_{n}\right] .
$$

Using the $\mathrm{SL}_{n}(\mathbb{Z})$ equivariance and translation invariance of $\mathrm{Z}$, we deduce that

$$
\mathrm{Z} \tilde{S}_{i}^{\prime}=a^{\prime}\left(S_{i}^{\prime}-\operatorname{cen}\left(S_{i}^{\prime}\right)\right)+b^{\prime}\left(-S_{i}^{\prime}+\operatorname{cen}\left(S_{i}^{\prime}\right)\right)+c_{0}\left[-e_{n}, e_{n}\right]
$$

for $i=1, \ldots, m$. As $\sum_{i=1}^{m} \mathrm{Z} \widetilde{S}_{i}^{\prime}=Q+c m\left[-e_{n}, e_{n}\right]$, we conclude that $c_{0}=c$. Thus (29) implies

$$
\mathrm{Z} \widetilde{T}_{n-1}=a^{\prime}\left(\widetilde{T}_{n-1}-\operatorname{cen}\left(\widetilde{T}_{n-1}\right)\right)+b^{\prime}\left(-\widetilde{T}_{n-1}+\operatorname{cen}\left(\widetilde{T}_{n-1}\right)\right) .
$$

To prove that $a=a^{\prime}$ and $b=b^{\prime}$, we first assume that $b \geq b^{\prime}$. Define the vector $v=e_{1}+\cdots+e_{n-1}$ and set $h_{0}=h\left(T_{n-1}-\operatorname{cen}\left(T_{n-1}\right), v\right)>0$. Note that

$$
h\left(-T_{n-1}+\operatorname{cen}\left(T_{n-1}\right), v\right)=(n-1) h_{0}
$$

and

$$
h\left(-\widetilde{T}_{n-1}+\operatorname{cen}\left(\widetilde{T}_{n-1}\right), v\right)=(n-1) h_{0} \text { and } h\left(\widetilde{T}_{n-1}-\operatorname{cen}\left(\widetilde{T}_{n-1}\right), v\right)=h_{0} .
$$

We consider the translation invariant real valued valuation $P \mapsto h(\mathrm{Z} P, v)$ on $\mathcal{P}\left(\mathbb{Z}^{n}\right)$ and for $k \geq 2$, the cell decomposition of $T_{n-1}+\left[o, k e_{n}\right]$ into $k$ translates of $\widetilde{T}_{n-1}$. The cell decomposition has $(k-1)$ faces intersecting the interior of $T_{n-1}+\left[o, k e_{n}\right]$, each a translate of $T_{n-1}$. Since $\operatorname{cen}\left(\mathrm{Z}\left(T_{n-1}+\left[o, k e_{n}\right]\right)\right)=o$ by Lemma 45, we have $h\left(\mathrm{Z}\left(T_{n-1}+\left[o, k e_{n}\right]\right), v\right) \geq 0$. By first applying the inclusion-exclusion principle (Corollary 15), second that $\mathrm{ZT}_{n-1}=\mathrm{Z}_{a, b} T_{n-1}$ and (32), and third that $a^{\prime}-a=b-b^{\prime}$, which follows from (29), we deduce that

$$
\begin{aligned}
0 & \leq h\left(\mathrm{Z}\left(T_{n-1}+\left[o, k e_{n}\right]\right), v\right) \\
& =k h\left(\mathrm{Z} \widetilde{T}_{n-1}, v\right)-(k-1) h\left(\mathrm{Z} T_{n-1}, v\right) \\
& =\left(k a^{\prime}+k(n-1) b^{\prime}-(k-1) a-(k-1)(n-1) b\right) h_{0} \\
& =\left(a^{\prime}+(n-1) b^{\prime}-(k-1)(n-2)\left(b-b^{\prime}\right)\right) h_{0} .
\end{aligned}
$$


As $b \geq b^{\prime}$ and the last expression is non-negative for any large $k$, we conclude that $b=b^{\prime}$. In turn, $a=a^{\prime}$ follows from (29).

If $b \leq b^{\prime}$, and hence $a \geq a^{\prime}$, we use essentially the same argument, only the valuation $P \mapsto h(\mathrm{Z} P,-v)$ replaces $P \mapsto h(\mathrm{Z} P, v)$, and we exchange the role of $a$ and $b$.

We deduce from Lemma 46 the following result.

Corollary 47. Let $\mathrm{Z}$ and the constants $a, b$ be as in Lemma 46. If $S_{1}, \ldots, S_{n}$ with $S_{1}=T_{n}$ are basic simplices triangulating $T_{n-1}+\left[0, e_{n}\right]$, then

$$
\sum_{i=1}^{n} \mathrm{Z}_{a, b} S_{i}=\sum_{i=1}^{n} \mathrm{Z} S_{i}
$$

\subsection{The faces of $\mathrm{Z} \mathrm{T}_{\mathrm{n}}$}

Let $n \geq 3$. Let $\mathrm{Z}: \mathcal{P}\left(\mathbb{Z}^{n}\right) \rightarrow \mathcal{K}\left(\mathbb{R}^{n}\right)$ be an $\mathrm{SL}_{n}(\mathbb{Z})$ equivariant and translation invariant Minkowski valuation and assume that Theorem 4 holds in $\mathbb{R}^{n-1}$. Hence, there exist $a, b \geq 0$ such that

$$
\mathrm{Z} P=\mathrm{Z}_{a, b} P
$$

for every lower dimensional $P \in \mathcal{P}\left(\mathbb{Z}^{n}\right)$. Note that Proposition 43 implies that $2 c=a+b$.

If $a+b=0$, then $\mathrm{Z}$ is simple. Hence, Lemma 41 implies that $\mathrm{Z} P=\{o\}$ for $P \in \mathcal{P}\left(\mathbb{Z}^{n}\right)$. Thus the proof of Theorem 4 is complete in this case.

Lemma 48. If $a+b>0$, then $\mathrm{Z} T_{n}$ is an $n$-dimensional polytope with the property that any of its facet normals is also a facet normal of $T_{n}-T_{n}$.

Proof. We use the triangulation $S_{1}, \ldots, S_{n !}$ of $[0,1]^{n}$ into basic simplices given by Lemma 16 with $S_{1}=T_{n}$. Write $\mathcal{F}^{\prime}$ for the faces of the cell decomposition that intersect the interior of $[0,1]^{n}$ and have dimension at most $n-1$. We deduce from the inclusion-exclusion principle that

$$
\mathrm{Z}[0,1]^{n}+\sum_{\substack{F \in \mathcal{F}^{\prime} \\ n-\operatorname{dim}(F) \text { odd }}} \mathrm{Z} F=\sum_{i=1}^{n !} \mathrm{Z} S_{i}+\sum_{\substack{F \in \mathcal{F}^{\prime} \\ n-\operatorname{dim}(F) \text { even }}} \mathrm{Z} F
$$


Note that (34) also holds for $\mathrm{Z}_{a, b}$ in place of $\mathrm{Z}$. Here $\mathrm{Z}_{a, b} F=\mathrm{Z} F$ for $F \in \mathcal{F}^{\prime}$ by (33). Therefore (34) combined with $a+b=2 c$ gives

$$
\sum_{i=1}^{n !} \mathrm{Z}_{a, b} S_{i}=\sum_{i=1}^{n !} \mathrm{Z} S_{i}
$$

We deduce from $a+b>0$ that the left hand side of (35) is $n$-dimensional. Since we have $S_{i}=\phi_{i} T_{n}$ with $\phi_{i} \in \mathrm{SL}_{n}(\mathbb{Z})$ and $\mathrm{Z} S_{i}=\phi_{i} \mathrm{Z} T_{n}$, it follows that $\mathrm{ZT}_{n} \neq\{o\}$. We deduce from Lemma 40 that $\mathrm{Z} T_{n}$ is $n$-dimensional.

We look at the decomposition of the unit cube $[0,1]^{n}$ into $T_{n}$ and the remaining part, $R_{n}$. Note that $T_{n} \cap R_{n}=\left[e_{1}, \ldots, e_{n}\right]$. Using Proposition 43 , (33), and the valuation property of $\mathrm{Z}$, we get

$$
\mathrm{Z} T_{n} \text { is a summand of } Q=[-c, c]^{n}+a\left[e_{1}, \ldots, e_{n}\right]-b\left[e_{1}, \ldots, e_{n}\right] \text {. }
$$

We deduce right away that $\mathrm{Z} T_{n}$ is a polytope.

Moreover, (2) and (36) imply that any facet normal of $\mathrm{ZT}_{n}$ is a facet normal of $Q$. Since $T_{n}-T_{n}$ is $O$-symmetric, (2) implies that it is now sufficient to show that the affine hull of any facet $F$ of $[-1,1]^{n}+\left[e_{1}, \ldots, e_{n}\right]-\left[e_{1}, \ldots, e_{n}\right]$ is parallel to a facet of $T_{n}-T_{n}$. Now $F=F_{0}+F_{1}-F_{2}$ where $F_{0}$ is a face of $[-1,1]^{n}$, and $F_{1}, F_{2}$ are faces of $\left[e_{1}, \ldots, e_{n}\right]$. In particular,

$$
d_{0}+d_{1}+d_{2} \geq n-1
$$

where $d_{i}=\operatorname{dim}\left(F_{i}\right)$. If $d_{0}=0$, then aff $F$ is a translate of aff $\left[e_{1}, \ldots, e_{n}\right]$ and hence aff $F$ is parallel to a facet of $T_{n}-T_{n}$. Therefore we may assume that $d_{0}>0$, and, without loss of generality, that aff $F_{0}$ is a translate of $\operatorname{span}\left\{e_{1}, \ldots, e_{d_{0}}\right\}$. Let $V_{i} \subset\left\{e_{1}, \ldots, e_{n}\right\}$ be the set of vertices of $F_{i}$ for $i=1,2$. Since

$$
d_{0}+\operatorname{card}\left(V_{1}\right)+\operatorname{card}\left(V_{2}\right)=d_{0}+d_{1}+d_{2}+2 \geq n+1,
$$

we have $\left\{e_{1}, \ldots, e_{d_{0}}\right\} \cap\left(V_{1} \cup V_{2}\right) \neq \emptyset$, where card stands for cardinality. Hence we may assume that $e_{1} \in V_{1}$ and $\left\{e_{1}, \ldots, e_{d_{0}}\right\} \cup V_{1}=\left\{e_{1}, \ldots, e_{m}\right\}$, where $m \leq d_{0}+d_{1}$. It follows from $e_{1} \in V_{1}$ that aff $F_{0}+$ aff $F_{1}$ is a translate of

$$
\operatorname{span}\left\{e_{i}: i=1, \ldots, d_{0}\right\}+\operatorname{span}\left\{e_{i}-e_{1}: e_{i} \in V_{1}\right\}=\operatorname{span}\left\{e_{i}: i=1, \ldots, m\right\} .
$$

Therefore aff $F$ is a translate of the affine hull of the facet $\left[o, e_{1}, \ldots, e_{m}\right]-F_{2}$ of $T_{n}-T_{n}$. 


\subsection{More on $\mathrm{Z} T_{n}$}

As in Section 2.8, set $T=T_{n}$ and $\alpha \bar{T}=T_{*}$. Define $Q_{*}=\alpha \mathrm{ZT}$. It follows from Lemma 48 that $Q_{*}$ is a polytope and that any of its facet normals is a positive multiple of $\sum_{i \in I} v_{i}$ for a proper subset $I$ of $\{0, \ldots, n\}$. Since $\mathrm{Z}$ is $\mathrm{SL}_{n}(\mathbb{Z})$ equivariant and translation invariant, $Q_{*}$ is invariant under maps from $\operatorname{Sym}\left(T_{*}\right)$. In particular, the orbits of the facet normals of $Q_{*}$ of this action are characterized by the cardinality of $I$.

Lemma 49. For $m=1, \ldots, n-2$, there exist constants $a_{m}, b_{m} \geq 0$ and $s_{m} \geq 0$ such that

$$
F\left(Q_{*}, w_{m}\right)=a_{m} F\left(T_{*}, w_{m}\right)+b_{m} F\left(-T_{*}, w_{m}\right)+s_{m} w_{m} .
$$

Proof. By Proposition 43 and (33), the decomposition (66) implies that

$$
Q_{*} \text { is a summand of }(a+b) \sum_{i=1}^{n}\left[o, v_{i}-v_{0}\right]+a\left[v_{1}, \ldots, v_{n}\right]-b\left[v_{1}, \ldots, v_{n}\right] \text {. }
$$

Let $m \in\{1, \ldots, n-2\}$. Since

$$
F\left(\sum_{i=1}^{n}\left[o, v_{i}-v_{0}\right], w_{m}\right)=\sum_{i=1}^{n} F\left(\left[o, v_{i}-v_{0}\right], w_{m}\right)=\sum_{i=1}^{m}\left[o, v_{i}-v_{0}\right],
$$

and

$$
F\left(\sum_{i=1}^{n}\left[o, v_{i}-v_{0}\right],-w_{m}\right)=\sum_{i=1}^{n} F\left(\left[o, v_{i}-v_{0}\right],-w_{m}\right)=\sum_{i=m+1}^{n}\left[o, v_{i}-v_{0}\right],
$$

we deduce from (12) and (37) that $F\left(Q_{*}, w_{m}\right)$ is a summand of

$$
(a+b) \sum_{i=1}^{m}\left[o, v_{i}-v_{0}\right]+a\left[v_{1}, \ldots, v_{m}\right]-b\left[v_{m+1}, \ldots, v_{n}\right]
$$

and that $F\left(Q_{*},-w_{m}\right)$ is a summand of

$$
(a+b) \sum_{i=m+1}^{n}\left[o, v_{i}-v_{0}\right]+a\left[v_{m+1}, \ldots, v_{n}\right]-b\left[v_{1}, \ldots, v_{m}\right] .
$$

Lemma 13 combined with (11) implies that

$$
F\left(Q_{*}, w_{m}\right)=L_{m}-b_{m} F\left(T_{*},-w_{m}\right)
$$


where $0 \leq b_{m} \leq b$ and $L_{m}$ is a convex polytope contained in a translate of $\operatorname{aff}\left(v_{0}, \ldots, v_{m}\right)$ and that

$$
F\left(Q_{*},-w_{k}\right)=a_{k}^{\prime} F\left(T_{*},-w_{k}\right)+L_{k}^{\prime}
$$

where $0 \leq a_{k}^{\prime} \leq a$ and $L_{k}^{\prime}$ is a convex polytope contained in a translate of aff $\left(v_{0}, \ldots, v_{k}\right)$. For $\rho \in \operatorname{Sym}\left(T_{*}\right)$ suitable and $k=n-m-1$, we have $\rho Q_{*}=Q_{*}$ and $\rho\left(-w_{k}\right)=w_{m}$. Hence (39) implies

$$
F\left(Q_{*}, w_{m}\right)=a_{n-m-1}^{\prime} F\left(T_{*}, w_{m}\right)+\rho L_{n-m-1}^{\prime}
$$

where $\rho L_{n-m-1}^{\prime}$ is contained in a translate of aff $\left(v_{m+1}, \ldots, v_{n}\right)$. Combining (38) and (40) with Lemma 13 shows that $F\left(Q_{*}, w_{m}\right)$ is a translate of $a_{m} F\left(T_{*}, w_{m}\right)+b_{m} F\left(-T_{*}, w_{m}\right)$ with $a_{m}=a_{n-m-1}^{\prime}$.

Thus there are $c_{0}, \ldots, c_{n} \in \mathbb{R}$ with $\sum_{i=0}^{n} c_{i}=1$ such that

$$
F\left(Q_{*}, w_{m}\right)=a_{m}\left[v_{0}, \ldots, v_{m}\right]-b_{m}\left[v_{m+1}, \ldots, v_{n}\right]+\sum_{i=0}^{n} c_{i} v_{i} .
$$

If $\rho \in \operatorname{Sym}\left(T_{*}\right)$ corresponds to an even permutation of $\left(v_{0}, \ldots, v_{m}\right)$ and $\left(v_{m+1}, \ldots, v_{n}\right)$, then $\rho Q_{*}=Q_{*}$ and $\rho w_{m}=w_{m}$. Hence (41) implies that

$$
\sum_{i=0}^{n} c_{i} \rho v_{i}=\sum_{i=0}^{n} c_{i} v_{i}
$$

This implies that $c_{0}=\cdots=c_{m}$ and $c_{m+1}=\cdots=c_{n}$. Thus $\sum_{i=0}^{n} c_{i} v_{i}=$ $\left(c_{0}-c_{n}\right) w_{m}$ and

$$
F\left(Q_{*}, w_{m}\right)=a_{m} F\left(T_{*}, w_{m}\right)-b_{m} F\left(T_{*},-w_{m}\right)+s_{m} w_{m}
$$

with $s_{m}=c_{0}-c_{n}$. If $a_{m}, b_{m}>0$, then $a_{m} F\left(T_{*}, w_{m}\right)-b_{m} F\left(T_{*},-w_{m}\right)$ is a facet of $a_{m} T_{*}-b_{m} T_{*}$. Thus (13) implies that $s_{m} \geq 0$. If $a_{m}=0$ or $b_{m}=0$, then also $s_{m} \geq 0$.

For $m=0, \ldots, n-1$, we set $G_{m}=F\left(Q_{*}, w_{m}\right)$. Then, for $m=1, \ldots, n-2$, we have

$$
G_{m}=a_{m}\left[v_{0}, \ldots, v_{m}\right]-b_{m}\left[v_{m+1}, \ldots, v_{n}\right]+s_{m} w_{m}
$$

If $a_{m}, b_{m}>0$, then $F\left(Q_{*}, w_{m}\right)$ is a facet of $Q_{*}$. Using (10), we obtain for its $(n-2)$-faces,

$$
\begin{aligned}
F\left(G_{m}, w_{m-1}\right) & =a_{m}\left[v_{0}, \ldots, v_{m-1}\right]-b_{m}\left[v_{m+1}, \ldots, v_{n}\right]+s_{m} w_{m} \\
F\left(G_{m}, w_{m+1}\right) & =a_{m}\left[v_{0}, \ldots, v_{m}\right]-b_{m}\left[v_{m+2}, \ldots, v_{n}\right]+s_{m} w_{m}
\end{aligned}
$$

We need the following result. 
Lemma 50. If $a_{m}, b_{m}>0$ for $m \in\{1, \ldots, n-2\}$, then $G_{m-1}$ and $G_{m+1}$ are facets of $Q_{*}$ and

$$
\begin{aligned}
& F\left(G_{m}, w_{m-1}\right)=F\left(Q_{*}, w_{m}\right) \cap F\left(Q_{*}, w_{m-1}\right) \\
& F\left(G_{m}, w_{m+1}\right)=F\left(Q_{*}, w_{m}\right) \cap F\left(Q_{*}, w_{m+1}\right) .
\end{aligned}
$$

Proof. We only consider the case of $F\left(G_{m}, w_{m+1}\right)$. Since $F\left(G_{m}, w_{m+1}\right)$ is an $(n-2)$-face of $Q_{*}$, we have

$$
F\left(G_{m}, w_{m+1}\right)=F\left(Q_{*}, w_{m}\right) \cap F\left(Q_{*}, v\right)
$$

where $v=\sum_{i \in I} v_{i}$ for a proper subset $I \subset\{0, \ldots, n\}$.

On the other hand, $v$ is orthogonal to the affine hull of $F\left(G_{m}, w_{m+1}\right)$. Therefore

$$
v=s w_{m}+t w_{m+1}=s\left(v_{0}+\cdots+v_{m}\right)-t\left(v_{m+2}+\cdots+v_{n}\right)
$$

for $s, t \in \mathbb{R}$. We deduce that $v \in\left\{ \pm w_{m}, \pm w_{m+1}, \pm v_{m+1}\right\}$. Readily $v \neq w_{m}$. Since $Q_{*}$ is $n$-dimensional, and $F\left(G_{m}, w_{m+1}\right) \subset F\left(Q_{*}, w_{m}\right)$, we have $v \neq$ $-w_{m}$. Next, $v \neq-v_{m+1}$ because $a_{m} v_{0}-b_{m} v_{m+2}+s_{m} w_{m} \in F\left(G_{m}, w_{m+1}\right)$ and $a_{m} v_{0}-b_{m} v_{m+1}+s_{m} w_{m} \in G_{m}$ but (10) implies that

$$
\begin{aligned}
h\left(Q_{*},-v_{m+1}\right) & \geq-v_{m+1} \cdot\left(a_{m} v_{0}-b_{m} v_{m+1}+s_{m} w_{m}\right) \\
& >-v_{m+1} \cdot\left(a_{m} v_{0}-b_{m} v_{m+2}+s_{m} w_{m}\right) .
\end{aligned}
$$

Next, $v \neq v_{m+1}$ because for $w=w_{m}-v_{m}+v_{m+1}=\sum_{i \in I} v_{i}$ corresponding to $I=\{0, \ldots, m-1, m+1\}$, we have $a_{m} v_{m+1}-b_{m} v_{m+2}+s_{m} w \in F\left(Q_{*}, w\right)$, and $s_{m} \geq 0$ gives

$$
\begin{aligned}
h\left(Q_{*}, v_{m+1}\right) & \geq v_{m+1} \cdot\left(a_{m} v_{m+1}-b_{m} v_{m+2}+s_{m}\left(w_{m}-v_{m}+v_{m+1}\right)\right) \\
& >v_{m+1} \cdot\left(a_{m} v_{0}-b_{m} v_{m+2}+s_{m} w_{m}\right) .
\end{aligned}
$$

Finally, $G_{m}=F\left(Q_{*}, w_{m}\right)$ is $(n-1)$-dimensional and $F\left(G_{m}, w_{m+1}\right)$ is $(n-2)$ dimensional. Thus

$$
h\left(Q_{*},-w_{m+1}\right) \geq h\left(G_{m},-w_{m+1}\right)>h\left(F\left(G_{m}, w_{m+1}\right),-w_{m+1}\right)
$$

and hence $v \neq-w_{m+1}$. Thus $v=w_{m+1}$. 
Proposition 51. For $n=3$, there exist $a_{0}, b_{0}, c_{0} \geq 0$ with the following properties. If $S$ is a basic three-dimensional simplex and $u, v \in \mathbb{R}^{3} \backslash\{o\}$ are such that $E=F(S, v)$ and $E^{\prime}=F(S,-v)$ are edges and $F=F(S, u)$ is a facet, then

$$
\begin{array}{rll}
F(\mathrm{Z} S, u) & \text { is a translate of } & a_{0} F+c_{0}(-F), \\
F(\mathrm{Z} S,-u) & \text { is a translate of } & b_{0}(-F)+c_{0} F, \\
F(\mathrm{Z} S, v) & \text { is a translate of } & a_{0} E+b_{0} E^{\prime} .
\end{array}
$$

In addition, $c_{0}=0$ if and only if $\mathrm{Z} S=\mathrm{Z}_{a_{0}, b_{0}} S$.

Proof. We may assume that $S=T_{3}$ and write $T=T_{3}$. If $\mathrm{Z} T=\{o\}$, then we have $a_{0}=b_{0}=c_{0}=0$. Otherwise, Lemma 40 implies that ZT is threedimensional. Note that by Lemma 48 the facet normals of $Q_{*}$ are a subset of $\left\{ \pm v_{i}: i=0,1,2,3\right\} \cup\left\{v_{i}+v_{j}: i \neq j\right\}$.

We claim that if $z$ is a vertex of $Q_{*}$, then

$$
z \in F\left(Q_{*}, v_{i}+v_{j}\right) \text { for some } i \neq j .
$$

To prove (42), we first assume that $a_{1}, b_{1}>0$. Then $F\left(Q_{*}, w_{1}\right)$ is twodimensional. If the vertex $z$ lies in $F\left(Q_{*}, v_{i}\right) \cap F\left(Q_{*}, v_{j}\right)$ for $i \neq j$, then

$$
F\left(Q_{*}, v_{i}+v_{j}\right) \subset \operatorname{aff} F\left(Q_{*}, v_{i}\right) \cap \operatorname{aff} F\left(Q_{*}, v_{j}\right) .
$$

This is not possible since $a_{1}, b_{1}>0$. Similarly, we see that for $i \neq j$ the vertex $z \notin F\left(Q_{*},-v_{i}\right) \cap F\left(Q_{*},-v_{j}\right)$. Since $z$ is contained in at least three two-dimensional faces of $Q_{*}, z \in F\left(Q_{*}, v_{i}+v_{j}\right)$ for some $i \neq j$.

Therefore we assume that either $a_{1}=0$ or $b_{1}=0$, that is, we have $\operatorname{dim} F\left(Q_{*}, w_{1}\right) \leq 1$. In this case, we deduce from Lemma 48 that any exterior normal to a two-dimensional face of $Q_{*}$ is an exterior normal to a two-dimensional face of either $T_{*}$ or $-T_{*}$. Hence

$$
Q_{*}=s T_{*} \cap\left(-t T_{*}\right) \text { for } s, t>0 .
$$

If $z$ is a vertex of $Q_{*}$, then it is not the midpoint of a segment contained in $Q_{*}$. Thus $z$ is contained in an edge of either $s T_{*}$ or $-t T_{*}$. Thus $z$ is a vertex of $F\left(Q_{*}, v_{i}+v_{j}\right)$ for some $i \neq j$, concluding the proof of (42).

Recall that $s_{1} \geq 0$ if $a_{1}+b_{1}>0$. In addition $s_{1}>0$ if $a_{1}=b_{1}=0$ as $o \in \operatorname{int} Q_{*}$. We deduce from Lemma 49 and (42), that

$$
Q_{*}=\left[a_{1} v_{i}-b_{1} v_{j}+s_{1}\left(v_{i}+v_{k}\right):\{i, j, k\} \subset\{0,1,2,3\}\right] .
$$


Thus it follows by a short calculation from (10) and (43) that

$$
\begin{aligned}
F\left(Q_{*},-v_{0}\right) & =a_{1}\left[v_{0}, v_{1}, v_{2}\right]-s_{1}\left[v_{0}, v_{1}, v_{2}\right]+\left(b_{1}+s_{1}\right)\left(v_{0}+v_{1}+v_{2}\right), \\
F\left(Q_{*}, v_{0}\right) & =-b_{1}\left[v_{1}, v_{2}, v_{3}\right]+s_{1}\left[v_{1}, v_{2}, v_{3}\right]+\left(a_{1}+s_{1}\right) v_{0}, \\
F\left(Q_{*}, w_{1}\right) & =a_{1}\left[v_{0}, v_{1}\right]-b_{1}\left[v_{2}, v_{3}\right]+s_{1}\left(v_{0}+v_{1}\right) .
\end{aligned}
$$

Therefore we may choose $a_{0}=a_{1}, b_{0}=b_{1}$ and $c_{0}=s_{1}$. Since $F\left(T_{*},-v_{0}\right)$ is a two-dimensional face and $F\left(T_{*}, \pm w_{1}\right)$ are edges, this concludes the proof.

\subsection{Proof of Theorem 4 for $\mathrm{n}=3$}

By Proposition 39, there exist $a, b \geq 0$ such that

$$
\mathrm{Z} P=\mathrm{Z}_{a, b} P
$$

for lower dimensional $P \in \mathcal{P}\left(\mathbb{Z}^{3}\right)$. Let $S_{1}, S_{2}, S_{3}$ with $S_{1}=T_{3}$ be the basic simplices triangulating the prism $\widetilde{T}_{2}=T_{2}+\left[0, e_{3}\right]$ defined in (17). Corollary 47 yields

$$
\mathrm{Z}_{a, b} S_{1}+\mathrm{Z}_{a, b} S_{2}+\mathrm{Z}_{a, b} S_{3}=\mathrm{Z} S_{1}+\mathrm{Z} S_{2}+\mathrm{Z} S_{3} .
$$

We observe that $F\left(S_{1},-e_{3}\right)=\left[o, e_{1}, e_{2}\right]$, that $F\left(S_{2},-e_{3}\right)=\left[e_{1}, e_{2}\right]$, that $F\left(S_{2}, e_{3}\right)$ is a translate of $\left[o, e_{1}\right]$ and that $F\left(S_{3}, e_{3}\right)$ is a translate of $\left[o, e_{1}, e_{2}\right]$. Set $G=F\left(\sum_{i=1}^{3} \mathrm{Z}_{a, b} S_{i},-e_{3}\right)$. Since $\left[o, e_{1}\right]$ is a translate of $\left[o,-e_{1}\right]$, we deduce that

$$
G \text { is a translate of } a\left[o, e_{1}, e_{2}\right]+b\left[o,-e_{1},-e_{2}\right]+a\left[e_{1}, e_{2}\right]+b\left[o, e_{1}\right] .
$$

By (44), we also have $G=F\left(\sum_{i=1}^{3} \mathrm{Z} S_{i},-e_{3}\right)$. Hence Proposition 51 implies that $G$ is a translate of

$$
\left(a_{0}+c_{0}\right)\left[o, e_{1}, e_{2}\right]+\left(b_{0}+c_{0}\right)\left[o,-e_{1},-e_{2}\right]+a_{0}\left[e_{1}, e_{2}\right]+b_{0}\left[o, e_{1}\right] .
$$

Hence $F\left(G,-e_{1}\right)$ is a translate of $a\left[o, e_{2}\right]$ by (45) and $F\left(G,-e_{1}\right)$ is a translate of $\left(a_{0}+c_{0}\right)\left[o, e_{2}\right]$ by (46). Thus $a=a_{0}+c_{0}$. From (45) and (46) we also obtain that $F\left(G, e_{1}\right)$ is a translate of $b\left[o,-e_{2}\right]$ and $F\left(G, e_{1}\right)$ is a translate of $\left(b_{0}+c_{0}\right)\left[o,-e_{2}\right]$, respectively. Hence $b=b_{0}+c_{0}$. Finally, we obtain that $F\left(G,-e_{2}\right)$ is a translate of $(a+b)\left[o, e_{1}\right]$ on the one hand, and is a translate of $\left(a_{0}+b_{0}+c_{0}\right)\left[o, e_{1}\right]$ on the other hand. Hence

$$
a_{0}+b_{0}+c_{0}=a+b=a_{0}+b_{0}+2 c_{0} .
$$

Therefore $c_{0}=0, a=a_{0}$ and $b=b_{0}$. Thus $\mathrm{ZT}_{3}=\mathrm{Z}_{a, b} T_{3}$ follows from Proposition 51. 


\subsection{Z $\mathrm{T}_{\mathrm{n}}$ for $\mathrm{n} \geq 4$}

Let $\mathrm{Z}$ be an $\mathrm{SL}_{n}(\mathbb{Z})$ equivariant and translation invariant Minkowski valuation on $\mathcal{P}\left(\mathbb{Z}^{n}\right)$. Let $n \geq 4$.

Proposition 52. If Theorem 4 holds in $\mathbb{R}^{n-1}$, then there exist $a_{0}, b_{0} \geq 0$ such that

$$
\mathrm{Z} S=\mathrm{Z}_{a_{0}, b_{0}} S
$$

for every basic $n$-simplex $S$.

Proof. Since $\mathrm{Z}$ is $\mathrm{SL}_{n}(\mathbb{Z})$ equivariant and translation invariant, it suffices to show there are $a_{0}, b_{0} \geq 0$ such that

$$
\mathrm{Z} T=\mathrm{Z}_{a_{0}, b_{0}} T
$$

where $T=T_{n}$. By Lemma 48, we may assume that Z $T$ is an $n$-dimensional polytope. As before we set $T_{*}=\alpha \bar{T}$ and $Q_{*}=\alpha \mathrm{ZT}$. Thus we have to show that that there are $a_{0}, b_{0} \geq 0$ such that

$$
Q_{*}=a_{0} T_{*}-b_{0} T_{*} .
$$

First, let $\operatorname{dim}\left(F\left(Q_{*}, w_{m}\right)\right) \leq n-2$ for $m=1, \ldots, n-2$.

Since Lemma 48 implies that $Q_{*}$ is a polytope whose facet normals are facet normals of $T_{*}-T_{*}$ and since $Q_{*}$ is invariant under the action of $\operatorname{Sym}\left(T_{*}\right)$, we deduce that there are $s, t>0$ such that

$$
Q_{*}=s T_{*} \cap\left(-t T_{*}\right) .
$$

We claim that either

$$
s \geq n t \text { or } t \geq n s,
$$

or in other words, either $-t T_{*} \subset s T_{*}$ or $s T_{*} \subset-t T_{*}$. Suppose that (49) does not hold, that is,

$$
\frac{s}{n}<t<n s .
$$

First, assume that $s \geq t$. Then $F\left(-t T_{*}, v_{0}+v_{1}\right)=\left[-t v_{2}, \ldots,-t v_{n}\right]$ satisfies

$$
\operatorname{cen}\left(F\left(-t T_{*}, v_{0}+v_{1}\right)\right)=t \frac{v_{0}+v_{1}}{n-1} \in t \operatorname{int} T_{*} \subset s \operatorname{int} T_{*} .
$$

Thus (48) implies that

$$
F\left(Q_{*}, v_{0}+v_{1}\right)=s T_{*} \cap F\left(-t T_{*}, v_{0}+v_{1}\right) \text { has dimension } n-2 .
$$


We have $a_{1}=0$ and $b_{1}>0$, and

$$
F\left(Q_{*}, v_{0}+v_{1}\right) \text { is a translate of } b_{1}\left[-v_{2}, \ldots,-v_{n}\right] \text {. }
$$

However, as $s<n t$, the vertices $-t v_{2}, \ldots,-t v_{n}$ of $F\left(-t T_{*}, v_{0}+v_{1}\right)$ are cut off by the facets $F\left(s T_{*},-v_{i}\right)$ of $s T_{*}$ for $i=2, \ldots, n$. Therefore (50) implies that $F\left(Q_{*}, v_{0}+v_{1}\right)$ has some $(n-3)$-dimensional faces with exterior normals $-v_{i}$ for $i=2, \ldots, n$. This contradicts (51), and in turn proves (49) if $s \geq t$. Finally, the case $s \leq t$ of (49) can be proved using the same argument for

$$
F\left(Q_{*},-v_{0}-v_{1}\right)=F\left(s T_{*},-v_{0}-v_{1}\right) \cap\left(-t T_{*}\right) .
$$

It follows from (49) that either $Q_{*}=s T_{*}$, or $Q_{*}=-t T_{*}$. Thus (47) holds in this case.

Second, let $F\left(Q_{*}, w_{m}\right)$ be $(n-1)$-dimensional for some $m=1, \ldots, n-2$. It follows from Lemma 50 that $\operatorname{dim}\left(F\left(Q_{*}, w_{k}\right)\right)=n-1$ for $k=0, \ldots, n-1$. It also follows from Lemma 50 that if $m=1, \ldots, n-2$, then

$$
\begin{aligned}
F\left(Q_{*}, w_{m-1}\right) & \cap F\left(Q_{*}, w_{m}\right) \\
& =a_{m}\left[v_{0}, \ldots, v_{m-1}\right]-b_{m}\left[v_{m+1}, \ldots, v_{n}\right]+s_{m} w_{m}, \\
F\left(Q_{*}, w_{m}\right) & \cap F\left(Q_{*}, w_{m+1}\right) \\
& =a_{m}\left[v_{0}, \ldots, v_{m}\right]-b_{m}\left[v_{m+2}, \ldots, v_{n}\right]+s_{m} w_{m} .
\end{aligned}
$$

If $m \geq 2$, then (53) applied to $F\left(Q_{*}, w_{m-1}\right) \cap F\left(Q_{*}, w_{m}\right)$ shows that

$$
\begin{aligned}
& F\left(Q_{*}, w_{m-1}\right) \cap F\left(Q_{*}, w_{m}\right) \\
& \quad=a_{m-1}\left[v_{0}, \ldots, v_{m-1}\right]-b_{m-1}\left[v_{m+1}, \ldots, v_{n}\right]+s_{m-1} w_{m-1}
\end{aligned}
$$

Comparing (52) and (54) implies $a_{m-1}=a_{m}, b_{m-1}=b_{m}$ and $s_{m}=s_{m-1}=0$.

Similar arguments based on (53) prove that if $m \leq n-3$, then $a_{m+1}=$ $a_{m}, b_{m+1}=b_{m}$ and $s_{m+1}=0$. Continuing step by step, we conclude that $a_{1}=\cdots=a_{n-1}, b_{1}=\cdots=b_{n-1}$ and $s_{1}=\cdots=s_{n-1}=0$.

Set $a_{0}=a_{1}$ and $b_{0}=b_{1}$. Since $s_{k}=0$ for $k=1, \ldots, n-1$, we obtain from Lemma 49 that

$$
F\left(Q_{*}, w_{k}\right)=F\left(a_{0} T_{*}-b_{0} T_{*}, w_{k}\right)
$$

for $k=1, \ldots, n-2$. It follows from Lemma 50 that

$$
h\left(Q_{*}, w_{k}\right)=h\left(a_{0} T_{*}-b_{0} T_{*}, w_{k}\right)
$$

for $k=0$ and $k=n-1$ as well. By symmetry, the support functions of $Q_{*}$ and $a_{0} T_{*}-b_{0} T_{*}$ agree for any possible facet normal of either polytope. Thus we conclude that (47) holds. 


\subsection{Proof of Theorem 4 for $\mathrm{n} \geq 4$}

Let $\mathrm{Z}: \mathcal{P}\left(\mathbb{Z}^{n}\right) \rightarrow \mathcal{K}\left(\mathbb{R}^{n}\right)$ be an $\mathrm{SL}_{n}(\mathbb{Z})$ equivariant and translation invariant Minkowski valuation. We prove Theorem 4 by induction on the dimension $n \geq 3$. The case $n=3$ is settled in Section 5.6. Therefore we assume that $n \geq 4$ and that Theorem 4 holds in dimension $(n-1)$. In particular, there exist $a, b \geq 0$ such that

$$
\mathrm{Z} P=\mathrm{Z}_{a, b} P
$$

for lower dimensional $P \in \mathcal{P}\left(\mathbb{Z}^{n}\right)$ (where $\mathrm{Z}_{a, b}$ is defined in (26) ). In addition, $a=b=0$ implies that $\mathrm{Z} P=\{o\}$ for $P \in \mathcal{P}\left(\mathbb{Z}^{n}\right)$, and $a+b>0$ implies that $\mathrm{Z} T_{n}$ is an $n$-dimensional polytope.

We may assume that $a+b>0$, and hence Proposition 52 implies the existence of $a_{0}, b_{0} \geq 0$ with $a_{0}+b_{0}>0$ such that if $S$ is a basic $n$-simplex, then

$$
\mathrm{Z} S=\mathrm{Z}_{a_{0}, b_{0}} S
$$

We compare $\mathrm{Z}$ and $\mathrm{Z}_{a, b}$.

Let $S_{1}, \ldots, S_{n}$ with $S_{1}=T_{n}$ be the basic simplices triangulating the prism $\widetilde{T}_{n-1}=T_{n-1}+\left[0, e_{n}\right]$ in (7). Corollary 47 implies that

$$
\sum_{i=1}^{n} \mathrm{Z}_{a, b} S_{i}=\sum_{i=1}^{n} \mathrm{Z} S_{i} .
$$

Let $1>r_{3}>\cdots>r_{n-1}>0$ and

$$
w=-e_{n}+e_{1}+e_{2}+\sum_{i=3}^{n-1} r_{i} e_{i} .
$$

It follows that $F\left(S_{i},-w\right)=\left\{e_{n}\right\}$ for $i=1, \ldots, n$ and that $F\left(S_{1}, w\right)=\left[e_{1}, e_{2}\right]$, $F\left(S_{2}, w\right)=\left[e_{1}, e_{2}\right]$, and $F\left(S_{i}, w\right)=\left\{e_{i-1}\right\}$ for $i=3, \ldots, n$. We deduce from the definition of $\mathrm{Z}_{a, b}$ and (55) that

$$
\begin{gathered}
F\left(\sum_{i=1}^{n} \mathrm{Z}_{a, b} S_{i}, w\right) \text { is a translate of } 2 a\left[e_{1}, e_{2}\right], \\
F\left(\sum_{i=1}^{n} \mathrm{Z} S_{i}, w\right) \text { is a translate of } 2 a_{0}\left[e_{1}, e_{2}\right],
\end{gathered}
$$


and hence $a=a_{0}$ follows from (156). Similarly

$$
\begin{gathered}
F\left(\sum_{i=1}^{n} \mathrm{Z}_{a, b} S_{i},-w\right) \text { is a translate of } 2 b\left[e_{1}, e_{2}\right], \\
F\left(\sum_{i=1}^{n} \mathrm{Z} S_{i},-w\right) \text { is a translate of } 2 b_{0}\left[e_{1}, e_{2}\right],
\end{gathered}
$$

and hence $b=b_{0}$. Therefore Corollary 18 implies that $\mathrm{Z}=\mathrm{Z}_{a, b}$ on $\mathcal{P}\left(\mathbb{Z}^{n}\right)$.

\subsection{Proof of Theorem 9}

Set $m_{n}=\operatorname{LCM}(2, \ldots, n+1)$. Proposition 28 implies that $m_{n} \ell_{1}(P) \in \mathbb{Z}^{n}$ for $P \in \mathcal{P}\left(\mathbb{Z}^{n}\right)$. Hence, for integers $a, b \geq 0$ with $b-a \in m_{n} \mathbb{Z}$, the operator $\mathrm{Z}$ defined by

$$
P \mapsto a\left(P-\ell_{1}(P)\right)+b\left(-P+\ell_{1}(P)\right)
$$

maps $\mathcal{P}\left(\mathbb{Z}^{n}\right)$ to $\mathcal{P}\left(\mathbb{Z}^{n}\right)$. For the reverse direction, let $\mathrm{Z}: \mathcal{P}\left(\mathbb{Z}^{n}\right) \rightarrow \mathcal{P}\left(\mathbb{Z}^{n}\right)$ be an $\mathrm{SL}_{n}(\mathbb{Z})$ equivariant and translation invariant Minkowski valuation. By Theorem 11, we know that there are $a, b \geq 0$ such that

$$
\mathrm{Z} P=a\left(P-\ell_{1}(P)\right)+b\left(-P+\ell_{1}(P)\right)
$$

for every $P \in \mathcal{P}\left(\mathbb{Z}^{n}\right)$. Since $\mathrm{Z} P \in \mathcal{P}\left(\mathbb{Z}^{n}\right)$ for all $P \in \mathcal{P}\left(\mathbb{Z}^{n}\right)$, setting $P=T_{k}$ and using Proposition 28 shows that

$$
a\left(T_{k}-\frac{e_{1}+\cdots+e_{k}}{k+1}\right)+b\left(-T_{k}+\frac{e_{1}+\cdots+e_{k}}{k+1}\right) \in \mathcal{P}\left(\mathbb{Z}^{n}\right) .
$$

Hence $a+c /(k+1),-(a+c)+c /(k+1) \in \mathbb{Z}$ for $k=1, \ldots, n$ with $c=b-a$. Thus $c=b-a \in m_{n} \mathbb{Z}$ and $a, b \in \mathbb{Z}$.

\section{Acknowledgments}

The authors thank Raman Sanyal for pointing out Corollary 23 and its proof to them, Peter McMullen for an improvement of the proof of Lemma 38 and Imre Bárány and Pál Hegedüs for valuable discussions. They also thank the referees for their helpful remarks.

The work of Károly J. Böröczky was supported, in part, by the Hungarian Scientific Research Funds No 109789 and No 116451. The work of Monika Ludwig was supported, in part, by Austrian Science Fund (FWF) Project P25515-N25. 


\section{References}

[1] J. Abardia, Difference bodies in complex vector spaces, J. Funct. Anal. 263 (2012), $3588-3603$.

[2] J. Abardia, Minkowski valuations in a 2-dimensional complex vector space, Int. Math. Res. Not. 5 (2015), 1247-1262.

[3] J. Abardia and A. Bernig, Projection bodies in complex vector spaces, Adv. Math. 227 (2011), 830-846.

[4] S. Alesker, Continuous rotation invariant valuations on convex sets, Ann. of Math. (2) 149 (1999), 977-1005.

[5] S. Alesker, Description of translation invariant valuations on convex sets with solution of P. McMullen's conjecture, Geom. Funct. Anal. 11 (2001), 244-272.

[6] S. Alesker and D. Faifman, Convex valuations invariant under the Lorentz group, J. Differential Geom. 98 (2014), 183-236.

[7] A. Barvinok, Integer points in polyhedra, Zurich Lectures in Advanced Mathematics, European Mathematical Society (EMS), Zürich, 2008.

[8] M. Beck and S. Robins, Computing the Continuous Discretely, Undergraduate Texts in Mathematics, Springer, New York, 2007.

[9] A. Bernig and J.H. G. Fu, Hermitian integral geometry, Ann. of Math. (2) 173 (2011), 907-945.

[10] U. Betke, Gitterpunkte und Gitterpunktfunktionale, Habilitationsschrift, Universität Siegen, 1979.

[11] U. Betke and M. Kneser, Zerlegungen und Bewertungen von Gitterpolytopen, J. Reine Angew. Math. 358 (1985), 202-208.

[12] K. J. Böröczky, Stronger versions of the Orlicz-Petty projection inequality, J. Differential Geom. 95 (2013), 215-247.

[13] S. Campi and P. Gronchi, The $L^{p}$-Busemann-Petty centroid inequality, Adv. Math. 167 (2002), 128-141.

[14] J. De Loera, J. Rambau, and F. Santos, Triangulations, Algorithms and Computation in Mathematics, vol. 25, Springer-Verlag, Berlin, 2010.

[15] E. Ehrhart, Sur les polyèdres rationnels homothétiques à $n$ dimensions, C. R. Acad. Sci. Paris 254 (1962), 616-618.

[16] R. Gardner, Geometric Tomography, second ed., Encyclopedia of Mathematics and its Applications, vol. 58, Cambridge University Press, Cambridge, 2006.

[17] P. M. Gruber, Convex and Discrete Geometry, Grundlehren der Mathematischen Wissenschaften, vol. 336, Springer, Berlin, 2007.

[18] C. Haberl, Blaschke valuations, Amer. J. Math. 133 (2011), 717-751. 
[19] C. Haberl, Minkowski valuations intertwining with the special linear group, J. Eur. Math. Soc. (JEMS) 14 (2012), 1565-1597.

[20] C. Haberl and L. Parapatits, The centro-affine Hadwiger theorem, J. Amer. Math. Soc. 27 (2014), 685-705.

[21] C. Haberl and L. Parapatits, Valuations and surface area measures, J. Reine Angew. Math. 687 (2014), 225-245.

[22] C. Haberl and F. Schuster, General $L_{p}$ affine isoperimetric inequalities, J. Differential Geom. 83 (2009), 1-26.

[23] H. Hadwiger, Vorlesungen über Inhalt, Oberfläche und Isoperimetrie, Springer, Berlin, 1957.

[24] A. Hatcher, Algebraic Topology, Cambridge University Press, Cambridge, 2002.

[25] D. A. Klain, A short proof of Hadwiger's characterization theorem, Mathematika 42 (1995), 329-339.

[26] D. A. Klain and G.-C. Rota, Introduction to Geometric Probability, Cambridge University Press, Cambridge, 1997.

[27] J. Li, S. Yuan, and G. Leng, $L_{p}$-Blaschke valuations, Trans. Amer. Math. Soc. 367 (2015), 3161-3187.

[28] M. Ludwig, Projection bodies and valuations, Adv. Math. 172 (2002), 158-168.

[29] M. Ludwig, Ellipsoids and matrix valued valuations, Duke Math. J. 119 (2003), 159188.

[30] M. Ludwig, Minkowski valuations, Trans. Amer. Math. Soc. 357 (2005), 4191-4213.

[31] M. Ludwig, Minkowski areas and valuations, J. Differential Geom. 86 (2010), 133161.

[32] M. Ludwig and M. Reitzner, A characterization of affine surface area, Adv. Math. 147 (1999), 138-172.

[33] M. Ludwig and M. Reitzner, A classification of SL( $n)$ invariant valuations, Ann. of Math. (2) 172 (2010), 1219-1267.

[34] E. Lutwak, D. Yang, and G. Zhang, $L_{p}$ affine isoperimetric inequalities, J. Differential Geom. 56 (2000), 111-132.

[35] E. Lutwak, D. Yang, and G. Zhang, Moment-entropy inequalities, Ann. Probab. 32 (2004), 757-774.

[36] E. Lutwak, D. Yang, and G. Zhang, Orlicz centroid bodies, J. Differential Geom. 84 (2010), 365-387.

[37] E. Lutwak, D. Yang, and G. Zhang, Orlicz projection bodies, Adv. Math. 223 (2010), $220-242$.

[38] E. Lutwak and G. Zhang, Blaschke-Santaló inequalities, J. Differential Geom. 47 (1997), 1-16. 
[39] P. McMullen, Valuations and Euler-type relations on certain classes of convex polytopes, Proc. London Math. Soc. (3) 35 (1977), 113-135.

[40] P. McMullen, Valuations and dissections, Handbook of Convex Geometry, Vol. B (P.M. Gruber and J.M. Wills, eds.), North-Holland, Amsterdam, 1993, 933-990.

[41] P. McMullen, Valuations on lattice polytopes, Adv. Math. 220 (2009), 303-323.

[42] P. McMullen and R. Schneider, Valuations on convex bodies, Convexity and its Applications (P.M. Gruber and J.M. Wills, eds.), Birkhäuser, 1983, 170-247.

[43] L. Parapatits, $S L(n)$-contravariant $L_{p}$-Minkowski valuations, Trans. Amer. Math. Soc. 366 (2014), 1195-1211.

[44] L. Parapatits, $S L(n)$-covariant $L_{p}$-Minkowski valuations, J. Lond. Math. Soc. 89 (2014), 397-414.

[45] L. Parapatits and T. Wannerer, On the inverse Klain map, Duke Math. J. 162 (2013), 1895-1922.

[46] R. Schneider, On Steiner points of convex bodies, Israel J. Math. 9 (1971), 241-249.

[47] R. Schneider, Krümmungsschwerpunkte konvexer Körper. II, Abh. Math. Sem. Univ. Hamburg 37 (1972), 204-217.

[48] R. Schneider, Convex Bodies: the Brunn-Minkowski Theory, expanded ed., Encyclopedia of Mathematics and its Applications, vol. 151, Cambridge University Press, Cambridge, 2014.

[49] F. Schuster, Crofton measures and Minkowski valuations, Duke Math. J. 154 (2010), $1-30$.

[50] F. Schuster and T. Wannerer, GL $(n)$ contravariant Minkowski valuations, Trans. Amer. Math. Soc. 364 (2012), 815-826.

[51] T. Wannerer, GL(n) equivariant Minkowski valuations, Indiana Univ. Math. J. 60 (2011), 1655-1672.

[52] T. Wannerer, The module of unitarily invariant area measures, J. Differential Geom. 96 (2014), 141-182.

Károly J. Böröczky

Alfréd Rényi Institute of Mathematics

Hungarian Academy of Sciences

1053 Budapest, Reáltanoda u. 13-15

Hungary

E-mail: carlos@renyi.hu

Monika Ludwig

Institut für Diskrete Mathematik und Geometrie

Technische Universität Wien

Wiedner Hauptstraße 8-10/1046

1040 Wien, Austria

E-mail: monika.ludwig@tuwien.ac.at 